"graumann" — 2007/8/21 — 13:22 — page 251 — \#1

\title{
Problemorientierung im Mathematikunterricht - ein Gesichtspunkt der Qualitätssteigerung
}

\author{
Günter Graumann und ERkKi Pehkonen
}

\begin{abstract}
The aim of this article is to give a synopsis of problem orientation in mathematics education and to stimulate the discussion of the development and research about problem-orientated mathematics teaching. At the beginning we present historical viewpoints of problem orientation and their connection with recent theories of cognition (constructivism). Secondly we give characterizations of concepts that stand in the context of problem-orientation and discuss different forms of working with open problems in mathematics teaching. Arguments for more problem orientation in mathematics education will be discussed afterwards. Since experience shows that the implementation of open problems in classroom produces barriers, we then discuss mathematical beliefs and their role in mathematical learning and teaching. A list of literature at the end is not only for references but also can be used to further research.

Zusammenfassung. Ziel des Beitrags ist es, eine Synopsis in Bezug auf Problemorientierung im Mathematikunterricht zu geben und die Diskussion bezüglich Entwicklung und Forschung eines problemorientierten Mathematikunterrichts zu stimulieren. Als Erstes werden historische Gesichtspunkte von Problemorientierung und deren Verknüpfung mit neueren Erkenntnistheorien (Konstruktivismus) vorgestellt. Zweitens werden Erläuterungen zu Begriffen, die im Kontext von Problemorientierung stehen, gegeben und verschiedene Ausprägungen der Behandlung offener Probleme im Mathematikunterricht diskutiert. Argumente für eine stärkere Berücksichtigung von Problemorientierung im Mathematikunterricht werden danach erörtert. Auf Barrieren bei der Implementierung von offenen Problemen im Unterricht, die durch mathematische Beliefs (Vorstellungen, Überzeugungen) geprägt sind, wird zum Schluss eingegangen. Die abschließend aufgeführte Literaturliste dient nicht nur dem Beleg der Zitate, sondern kann auch zu weiterer Vertiefung genutzt werden.
\end{abstract}

Key words and phrases: mathematics teaching, problem solving, historical notes on problem solving.

ZDM Subject Classification: A30, D40, D50.

Copyright (C) 2007 by University of Debrecen 


$$
\text { "graumann" — 2007/8/21 — 13:22 — page } 252 \text { — \#2 }
$$

Die Forderung nach Problemorientierung im Unterricht und mehr Selbsttätigkeit der Lernenden hört man heutzutage immer häufiger; solche expliziten Forderungen gibt es aber schon seit über hundert Jahren. In den 1980er Jahren avancierte Problemlösen (problem solving) innerhalb der Mathematikdidaktik dann sogar zu einer Leitidee für den Mathematikunterricht (vgl. Krulik \& Reys 1980, Zimmermann 1983) und seit den 1990er Jahren wird international Problemorientierung in der Form von Erkundungen (investigations) und offenen Problemen (open problems) (vgl. etwa Pehkonen 2001a und Kapitel 4) propagiert. Ende der 1990er Jahre haben die Autoren die inzwischen internationale Forschergruppe „Promath“ gegründet, die sich zur Aufgabe gemacht hat, Problemorientierung zu fördern und insbesondere Möglichkeiten und Grenzen von Problemorientierung im Mathematikunterricht zu erforschen (vgl. Veistinen 2002, Rehlich \& Zimmerman 2004). Die alltägliche Unterrichtspraxis war und ist jedoch immer noch weit entfernt von solchen Vorstellungen. Deutlich wird dies etwa durch die Ergebnisse von TIMSS - insbesondere die in Deutschland, Japan und den USA durchgeführten Videostudien - und PISA (vgl. z.B. Neubrand \& Neubrand 1998, Deutsches PISAKonsortium 2001).

Unser Ziel hier ist es nun, mittels einer Klärung der betreffenden Begrifflichkeit, einer Synopsis verschiedener Richtungen (einschließlich deren Einbettung in historische Entwicklungen) sowie der Darlegung möglicher Widerstände bei der Implementierung einer veränderten Unterrichtskultur die Diskussion über die Forschung und Entwicklung zu Problemorientierung im Mathematikunterricht erneut anzuregen und zu differenzieren, wobei wir die Beschäftigung mit offenen Problemen bzw. Problemfeldern als besonders wichtig ansehen.

Erwähnt sei auch, dass mit dem Begriff „Problemorientierung“ keine Ausprägungen, die im Folgenden näher charakterisiert werden.

\section{Problemorientierung und Entdeckendes Lernen in den letzten hundert Jahren}

Ein Blick in die Geschichte der Pädagogik im Allgemeinen und des Mathematikunterrichts im Besonderen zeigt, dass - wie schon erwähnt - die heutigen Bestrebungen bezüglich Problemorientierung durchaus nicht neu und original sind. Die Bedeutung von selbsttätigem bzw. gelenktem Entdecken als Lernmethode wurde seit Sokrates immer wieder hervorgehoben (vgl. etwa Foster 1974, S. 23). In der Neuzeit sind hier vor allem Rousseau und Fröbel zu nennen. Rousseau 


$$
\text { "graumann" — 2007/8/21 — 13:22 — page } 253 \text { — \#3 }
$$

sieht als erster im Kind ein Wesen der Natur, dessen Kräfte zur Selbstentfaltung gefördert werden müssen und Fröbel entwickelt Materialien (z.B. den zweifach geteilten Würfel), an denen die Kinder durch eigentätige Handlungen Erkenntnisse gewinnen sollen.

Seit Ende des 19. Jahrhunderts - im Rahmen der Reformpädagogik - findet man dann mehrfach Forderungen nach selbsttätigem und aktivem Lernen. Dabei werden unterschiedliche Begriffe wie „entdeckendes Lernen“, „Lernen durch Erfahrung", „aktives Lernen“ oder „fragend-forschender Unterricht" verwendet. Häufig wird aber auch einfach nur von „Problemlösen im Unterricht“ oder „problem-orientierter Unterricht“ gesprochen. Jeder einzelne Begriff ist zwar mit unterschiedlichen Akzentuierungen verbunden, für unsere Zwecke brauchen diese aber nicht näher analysiert zu werden; wir wollen lediglich einige Charakteristika, die auch heute noch von Bedeutung sind, beschreiben.

Selbsttätiges Lernen als Unterrichtsprinzip wurde zuerst vor gut hundert Jahren - vor allem von C. Mason, R. MacMillan, M. MacMillan und M. Montessori - gefordert ${ }^{1}$. Zur gleichen Zeit wurde die amerikanische Pädagogik entscheidend von J. Dewey $(1949)^{2}$ geprägt. Er favorisierte das ,learning by doing " und hat damit zur Verbreitung der Ideen des selbsttätigen und aktiven Lernens in Nordamerika wesentlich beigetragen. „In seinen pädagogischen Schriften, besonders in Demokratie und Erziehung (1949), stellt er die Anregung des Denkens als eine Hauptfunktion der Erziehung heraus. Die Ziele der Erziehung können, so behauptet er, am besten durch ,Erfahrung' erreicht werden" (Berlyne 1973, S. 96$)^{3}$.

In England spielte Selbsttätigkeit und Entdeckung während des ganzen 20. Jahrhunderts eine wichtige Rolle. Erwähnt seien hier etwa A. S. Neill mit seinem schon in den 20er Jahren entwickelten Konzept von Summerhill sowie S. Isaacs

${ }^{1}$ „Beispiele für entdeckendes Lernen lassen sich in jeder Epoche auffinden. Die großen Pädagogen sind immer für das Prinzip des eigenen Entdeckens eingetreten und haben in der Erziehung ein auf Selbsttätigkeit beruhendes Vorgehen befürwortet. Doch erst wenn wir die Entwicklung unseres Schulsystems in den letzten hundert Jahren betrachten, können wir sehen, wie sich auch im Unterricht mit Schülergruppen das entdeckende Lernen als klare Linie herausbildet. Die Arbeiten von Charlotte Mason, Rachel und Margret MacMillan und Maria Montessori zeigen, daß aufgeklärte, fortschrittliche Pädagogen schon im vergangenen Jahrhundert eine Richtung verfolgt haben, deren Ziel das eigenständige Tun der Kinder war. Sie entwickelten Systeme, bei denen Kinder lernen konnten, indem sie selbst Lösungswege herausfanden" (Foster 1974, S. 24).

2 Jahreszahl der deutschen Ausgabe, Originalausgabe 1915.

${ }^{3}$ Hieran knüpft auch H. v. Hentig mit seinen Konzept für die Laborschule in Bielefeld an (vgl. z.B. sein Buch „Schule als Erfahrungsraum“, Stuttgart 1973). 
"graumann" — 2007/8/21 — 13:22 — page 254 — \#4

(1932, 1948), die Untersuchungen über das Niveau der geistigen Entwicklung von Kindern bei reicher Anregung gemacht hat ${ }^{4}$.

Auch in Deutschland wurde von Reformpädagogen die Bedeutung der Tätigkeit der Lernenden betont. Erwähnt sei hier etwa H. Gaudig, der insbesondere die Notwendigkeit der Förderung der Frageaktivität der Lernenden betont. „Beim Kinde ist die Frage das Mittel, seinen Wissensdrang zu befriedigen; mit der Frage pocht es an die Pforten der Erkenntnis und Weisheit. [...] Wer fragen kann, vermag sich oder anderen Denkaufgaben zu stellen. Die Kunst ist umso wertvoller, je sinnvoller die Fragestellung ist. Die Frage aber wird umso sinnvoller, je mehr sie sich innerhalb von Gedankenzusammenhängen entwickelt und hier wieder, je mehr sie innerhalb solcher Zusammenhänge das Denken planmäßig in richtige Bahnen lenkt" (Gaudig 1930, S. 68-69). In ähnlicher Weise argumentiert auch F. Copei, der dem deskriptivem Unterricht den fragend-forschenden Unterricht, bei dem eine Problemspannung sich entfalten lässt, die in den Lernenden als Folge selbsterfahrener kognitiver Konflikte entsteht, gegenüber stellt. „Durch das sorgfältige methodische Wegräumen aller Hindernisse kommt es zu gar keiner wirklichen Spannung im Geiste des Kindes. So wird ihm das Ergebnis auch nicht wirkliche Lösung. [...] Das bleibt ein Ziel der intellektuellen Erziehung: nicht den Gegenstand durch ihn wesenfremde Zutaten ,interessant zu machen', sondern im Geist aus der Frage ein echtes ,Interesse' erwachsen zu lassen" (Copei 1950, S. 114 und 116).

Eine Betonung des Aspektes „Problemorientierung“ und eine genauere Untersuchung von Problemlöseprozessen geht auf die zwischen 1920 und 1945 durchgeführten Forschungen der Gestaltpsychologen (vgl. etwa Köhler 1925, Duncker 1935 und Wertheimer 1945) zurück. Die Gliederung von Problemlöseprozesse in bestimmte Phasen, die Verwendung heuristischer Strategien beim Prozess der Lösungsfindung und die Berücksichtung nicht-linearer Lernprozesse trat damit in das Blickfeld pädagogischer Überlegungen. Bemerkenswert ist, dass die Gestaltpsychologen viele Beispiele für ihre Untersuchungen dem Bereich der Elementarmathematik entnommen haben. Eine der ersten experimentellen Studien über das entdeckende Lernen (McConnel 1934) orientierte sich ebenfalls

4 „Im Verlauf der Jahre haben sich immer wieder von der Regierung in Auftrag gegebene Gutachten zum Wert des entdeckenden Lernens und der auf selbständiges Arbeiten abzielenden Methoden geäußert. Das bekannte Zitat aus dem Hadow Report (1931), daß man beim Aufstellen eines Lehrplans eher von Tätigkeiten und Erfahrungen ausgehen müsse als von Wissen, das erworben, und von Fakten, die gespeichert werden sollten - dieses Zitat ist ein Beweis für die Sympathie, die von offizieller Seite fortschrittlichen Methoden entgegengebracht wird. Viele Lehrer griffen die Anregung dieses Berichtes auf und führten sie weiter." (Foster 1974, S. 26). 


$$
\text { "graumann" — 2007/8/21 — 13:22 — page } 255 \text { — \#5 }
$$

Problemorientierung im Mathematikunterricht - ein Gesichtspunkt d. Qualitätssteigerung 255

an der gestalttheoretischen Lerntheorie (vgl. auch Neber 1973, S. 8). Erwähnt sei außerdem, dass in dem der Gestaltpsychologie nahestehenden ganzheitlichen Unterricht nach J. Wittmann (1929) ein problemorientiertes Vorgehen mit entdeckender Methodik eine nicht unbedeutende Rolle spielt. Die Auseinandersetzung mit Problemlösestrategien und Phaseneinteilungen des Problemlöseprozesses in der Mathematik und der Mathematikdidaktik wurde in den 1940er und 1950er Jahren sehr stark durch die ungarischen Mathematiker G. Polya (vgl. etwa seine Veröffentlichung „How to solve it" 1945 bzw. in Deutsch „Schule des Denkens", 1949) und J. Hadamard (,The Psychology of Invention in the Mathematical Field“ 1954) angeregt. Schon zu Beginn des 20. Jahrhunderts entstand in Ungarn eine Vereinigung von Mathematikern und Mathematiklehrern, die eine außerunterrichtliche Förderung mittels Problemlöseaktivitäten von mathematisch interessierten Schülern betrieb. Bis heute ist der Mathematikunterricht in Ungarn von diesen Gedanken geprägt. Außerdem ist eine Reihe berühmter Mathematiker aus dieser „Schule“ hervorgegangen (vgl. Vansco 2002).

Eine intensive Auseinandersetzung mit einer fast unüberschaubaren Zahl von Veröffentlichungen über verschiedene Formen des entdeckenden Lernens, Vor- und Nachteile verschiedener Unterrichtsformen und empirischer Untersuchungen setzte dann Ende der 40er Jahre des 20. Jahrhunderts vor allem im englischsprachigen Raum ein und erreichte dort einen Höhepunkt Anfang der 60er Jahre. Erwähnt seien hier nur die beiden bedeutendsten Vertreter J. Bruner $(1960,1961)$ und D. P. Ausubel (1961, 1968), die auch in Deutschland sehr bekannt wurden und bei uns vor allem in den 1970er Jahren eine Diskussion über Problemorientierung und entdeckendes Lernen anregten.

J. S. Bruner hat dabei den Begriff „Entdeckendes Lernen“ ( „learning by discovery ") zunächst benutzt, um die auf behavioristischen Lerntheorien basierten Unterrichtsmethoden zu überwinden. „Um die Dominanz des Reiz-ReaktionsModells zu überwinden, wurde von Bruner ,entdeckendes Lernen' gefordert. Lernen ist danach kein ausschließlich reaktiver und von außen gesteuerter Prozeß, sondern ein aktiver Konstruktionsvorgang. [...] Bei diesem Prozeß werden Informationen über Sachverhalte etwa durch aktives Fragen oder systematisches Beobachten gesucht und mit Hilfe des bereits vorhandenen Wissens sinnvoll verarbeitet. [...] Als ,entdeckendes Lernen' faßte Bruner daher alle ,Formen des Wissenserwerbs mit Hilfe des eigenen Verstandes' zusammen, die im Extrem ohne äußere Lenkung ablaufen." (Neber 1995, S. 512). 
"graumann" — 2007/8/21 — 13:22 — page 256 — \#6

In der Mathematikdidaktik der zweiten Hälfte des 20. Jahrhunderts haben die in der Pädagogik und Psychologie geführten Diskussionen über „Entdeckendes Lernen“ und „Problemorientierung“ verschiedene Aktivitäten ausgelöst, die vor allem in den 60er und 70er Jahren des 20. Jahrhunderts in den USA und in England zu einzelnen Forschungsprojekten und Unterrichtsversuchen sowie Curriculumvorschlägen führten. Das Hauptgewicht lag dabei jedoch nicht auf theoretischen Erörterungen, sondern auf der Erstellung und Erprobung von Aufgaben bzw. Aufgabensequenzen (vgl. etwa Polya 1962, 1965; Botts 1965, Kilpatrick 1969a, 1969b, Polya \& Kilpatrick 1974, Lockard 1975/77, Schoenfeld $1979)^{5}$.

Während der 1970er Jahre versuchte man die Problemlösefähigkeit von Schülern durch das direkte Unterrichten heuristischer Strategien (Heurismen) ${ }^{6} \mathrm{zu}$ erhöhen (vgl. z.B. Schoenfeld 1979). Wesentliche Resultate von Forschungen hierzu findet man in dem Buch „Mathematical Problem Solving“ (Schoenfeld 1985). U.a. stellt er dort fest, dass die Komponenten, welche ein Individuum beim Problemlösen benötigt, in vier Gruppen geteilt werden können: (1) Ressourcen, (2) Heurismen, (3) Kontrolle, (4) Belief-Systeme. In den 1980er Jahren wurde aber klar, dass das Lernen von heuristischen Strategien nicht allein für die Entwicklung der Problemlösefähigkeiten ausreicht (vgl. z.B. Schoenfeld 1983, Silver 1985), insbesondere muss man auch die Metakognitionen ${ }^{7}$ der Lernenden entwickeln, die u.a. die Wahl ihrer heuristischen Strategien lenken (vgl. Pehkonen 1991 und Kapitel 6). Weiterhin wurde deutlich, dass Problemlösen nicht als Unterrichtsthema, sondern als Unterrichtsmethode angesehen werden muss (Schröder \& Lester 1989).

Die Analyse des Problemlöseverhaltens von Mathematikerinnen und Mathematikern lenkte dann die Aufmerksamkeit darauf, dass bei der Bearbeitung von Problemen kreatives Verhalten von großer Bedeutung ist (vgl. etwa Schoenfeld 1985). Deshalb wurde neben der Forschung über Metakognitionen in den 1990er Jahren auch die Kreativität bei der Beschäftigung mit Mathematik näher

${ }^{5}$ Einen Einblick in britische Projekte kann man bei British Council \& Nuffield Foundation 1978 und Howson 1978 finden. Eine ausführliche Bibliographie der amerikanischen Projekte und Veröffentlichungen zu dieser Zeit findet man z.B. in Hill 1979, Krulik \& Reys 1980 und Schoenfeld 1983.

${ }^{6}$ Nach Schoenfeld (1985) kann man heuristische Strategien als Methoden, die ein Individuum beim Problemlösen benötigt, charakterisieren.

7 Nach Schoenfeld sind Metakognitionen Reflexionen über das eigene Denken. Forschungen hierüber fokussieren oft auf folgenden drei Kategorien: „1. Your knowledge about your own thought processes. .. 2. Control, or self-regulation. ... 3. Beliefs and intuitions." (Schoenfeld 1987, S. 190). 


$$
\text { "graumann" — 2007/8/21 — 13:22 — page } 257 \text { — \#7 }
$$

Problemorientierung im Mathematikunterricht - ein Gesichtspunkt d. Qualitätssteigerung 257

untersucht (vgl. z.B. Kieswetter 1977, Pehkonen 1997a, Silver 1997, Weth 1999, Zimmermann 1999b). Als einen Vorläufer dieser Forschungslinie in Deutschland kann man K. Kiesswetter betrachten. Er kombinierte schon vor etwa dreißig Jahren Untersuchungen zur Kreativität mit dem informationstheoretischen Ansatz des Problemlösens, indem er ein Modell des Problemlöseprozesses als Entwicklung eines Wissensgraphs darstellte (Kiesswetter 1977, 1983). Hierbei wurde klar, dass beim Lösen mathematischer Probleme sowohl mathematisches Wissen als auch Kreativität vonnöten ist und miteinander verbunden werden muss. Ebenfalls sind die Problemfindungsaktivitäten stark mit Kreativität verbunden. Fragen der Problemfindung und des Erstellens von Problemen werden u.a. seit etwa zwanzig Jahren von einer Gruppe um E. Silver untersucht (vgl. Silver 1995).

In Deutschland gab es in den 1960er und 1970er Jahren nur einzelne Beiträge über Problemorientierung im Mathematikunterricht und das Lehren und Lernen von heuristischen Strategien (vgl. etwa Denk 1964, Hürten 1971, 1976, Freudenthal 1973, Glatfeld 1977, Hinkfuß 1980). Sie wurden vermutlich angeregt durch die englisch-sprachige Literatur, die Diskussion in Deutschland über entdeckendes Lernen in der Pädagogik und in den naturwissenschaftlichen Fächern sowie die deutsch-sprachigen Veröffentlichungen von Polya über Heuristik und Plausibles Schließen (vgl. etwa Polya 1962/63 und 1964). Der größte Teil der deutschen Mathematikdidaktiker beschäftigte sich in dieser Zeit jedoch mit der Neuen Mathematik und deren Betonung mathematischer Strukturen. Als Reaktion hierauf entstand in der zweiten Hälfte der 70er Jahre eine Diskussion um Praxis- und Projektorientierung im Mathematikunterricht, bei denen problemorientierte Zugänge ebenfalls eine wichtige Rolle spielen (vgl. etwa Graumann 1976, 1977; Münzinger 977, Mued 1979). In den 1980er und 1990er Jahren wird die Bedeutung von „Problemorientierung im Mathematikunterricht" in der mathematikdidaktischen Diskussion in Deutschland zwar allgemein anerkannt; es gibt jedoch nur vereinzelte Veröffentlichungen mit praktischen Unterrichtsvorschlägen oder Analysen zu Erfahrungen mit Problemorientierung (insbesondere bezüglich Vorbereitungen zu Mathematikolympiaden oder ähnlichen Kursen). Seit den 1990er Jahren beschäftigen sich mit Problemfindungsaspekten im Rahmen von Problemfeldern mehrere Mathematikdidaktiker (vgl. etwa Zimmermann 1986, Graumann 1994, 1999, 2000, 2002, Schupp 1995, 1998, 2002, Hein \& Knichel 1999, Henning \& Leneke 2000) und zum Aspekt der Kreativität findet man - wie oben erwähnt - Ende der 1990er Jahre auch in Deutschland mathematikdidaktische Veröffentlichungen. In der alltäglichen Schulpraxis kommen Phasen eines problemorientierten Mathematikunterrichts jedoch immer noch selten vor. Erst 


$$
\text { "graumann" — 2007/8/21 — 13:22 — page } 258 \text { — \#8 }
$$

Ende der 1990er Jahre erscheinen erste Schulbücher mit einer stärkeren Betonung von inner- und außermathematischen Problemen und es werden in Lehrerfortbildungen (vgl. etwa www.learn-line.nrw.de/angebote/sinus/projekt3) Formen und Möglichkeiten selbstregulierten Lernens im Mathematikunterricht erörtert.

\section{Konstruktivismus und Mathematikunterricht}

Angeregt durch die Forschungen von Kognitionspsychologen über konstruktivistische Erkenntnistheorien erfolgte seit Anfang der 1980er Jahre, zuerst in den USA und später auch in Europa und Asien, eine Neubelebung der Diskussion von offenen Lernformen und problemorientiertem Arbeiten in der Mathematikdidaktik (vgl. etwa Schoenfeld 1985, 1987, Silver 1985, Pehkonen 1991), bei der jetzt auch theoretische Reflexionen mehr in den Vordergrund rückten.

Als Vorläufer des modernen Konstruktivismus werden Dewey, Vygotsky und vor allem Piaget genannt ${ }^{8}$. Ernst von Glasersfeld hat dann in den 1980er Jahren aufbauend auf Piagets Epistemologie den sogenannten „radikalen Konstruktivismus " geprägt (vgl. Glasersfeld 1991, 1996). Dabei handelt es sich um eine Theorie über den Erwerb von Wissen, nach der die Erkenntnis von einer objektiven Welt nicht möglich ist (obgleich die Existenz einer solchen Welt nicht prinzipiell abgelehnt wird). Jegliches Wissen ist nur eine Konstruktion von einzelnen Individuen. „Any attempt to test the truth of what is known must itself be an act of knowing and hence subjective. Any knowledge of 'objective truth', therefore, is impossible. Constructivism cuts the Gordian knot by separating epistemology from ontology. [...] Von Glasersfeld (1985) claims that constructivism 'deliberately and consequentially avoids saying anything about ontology" (Kilpatrick 1987, S. 6 und 18).

Das erworbene Wissen kann deshalb nicht danach beurteilt werden, ob es die Wirklichkeit treffend beschreibt oder nicht. Es gibt kein ,wahr' oder ,falsch' in diesem Sinne. Wissen kann nur danach beurteilt werden, ob es in Bezug auf bestimmte Kontexte, Interessen und Probleme hin geeignet ist, d. h. gute Erklärungen und

8 „It is primarily the influence of Jean Piaget which has established constructivism as a central philosophy in the psychology of mathematics education. His constructivism has a number of components including an epistomology, a structuralist view, and a research methodology although I do not claim that these are either exhaustive or independent." (Ernest 1991b, S. $25 / 26)$. 


$$
\text { "graumann" — 2007/8/21 — 13:22 — page } 259 \text { — \#9 }
$$

Problemorientierung im Mathematikunterricht - ein Gesichtspunkt d. Qualitätssteigerung 259

gutes Verständnis sowie tragbare Handlungsperspektiven bzw. Lösungen liefert. Die Konstruktivisten sprechen in diesem Zusammenhang von „Viabilität“.

Ergänzt wurde dieser Ansatz durch die Feststellung, dass der Mensch ein soziales Wesen ist, dessen Lernprozesse fast immer in einem sozialen Feld und einem Diskurs mit kommunikativen und interaktiven Aspekten stattfinden. Fehler und Widersprüche sowie deren Korrektur in der Auseinandersetzung mit der Umgebung sind dabei von zentraler Bedeutung. Die individuellen Konstruktionen sind stets in einer komplexen Lernsituation mit unterschiedlichen Einflussfaktoren eingebunden. Will man diesen Gesichtspunkt deutlich werden lassen, so spricht man vom „sozialen Konstruktivismus".

Seit Mitte der 1990er Jahre tauchen in deutschsprachigen Publikationen Begriffe wie „Konstruktivistische Didaktik“ und „Pädagogischer Konstruktivismus“ (vgl. Siebert 1994 und 1999, V. Glasersfeld 1995, Reich 1997, 1998 und Terhart 1999) auf. Es geht dabei um eine didaktische Umsetzung der Grundideen des Konstruktivismus (wobei nicht immer auf das Konzept des radikalen Konstruktivismus Bezug genommen wird, sondern oft nur Gesichtspunkte des sogenannten „trivialen Konstruktivismus“, wie er bei Piaget zu finden ist, als Basis dienen).

Als wesentliche Folgerungen für die Erzeugung von Lernprozessen kann festgehalten werden, dass eine Abbilddidaktik nicht erfolgreich sein kann und dass das Lernen aufgrund reiner verbaler oder visueller Präsentationen des Inhaltes nur aufgrund parallel oder nachträglich verlaufender aktiver Auseinandersetzung der Lernenden mit dem präsentierten Inhalt Erfolg haben kann. Die Aufgabe der Lehrenden sollte deshalb weniger in der Präsentation von Inhalten als vielmehr in der Organisation von geeigneten Angeboten zur selbständigen Auseinandersetzung der Lernenden mit dem Inhalt (die dem Entwicklungsstand der Lernenden angemessen und entsprechend vorstrukturiert sind) bestehen. Hierzu sind dann immer wieder auch sogenannte „Pertubationen“, d. h. notwendige Störungen zur Anregung von Konstruktionen, angebracht. In der Sprache von Piaget ausgedrückt heißt das, dass Situationen geschaffen werden müssen, die das innere Gleichgewicht stören und damit zu Akkomodationen anregen.

In Anlehnung an P. Ernest (1994) lassen sich Prozesse des Mathematiklernens - aus der Position des ,sozialen Konstruktivismus' - wie folgt skizzieren: „Beim Mathematiklernen wenden die Lernenden eigene Sinnkonstruktionen, die sie bis dato ausgebildet haben, auf die mathematische Handlungssituation an: Sie agieren, gesteuert durch eigene Sinnkonstruktionen, die ihnen im Moment passend erscheint. Gelingt - auch das ist zunächst subjektive Einschätzungssache - 


$$
\text { "graumann" — 2007/8/21 — 13:22 — page } 260 \text { — \#10 }
$$

die Handlung, so fühlen sich die Lernenden in ihrer Sinnkonstruktion bestätigt. Gelingt sie nicht, trifft sie also auf,Widerstände', so revidieren die Lernenden ihre jeweilige Sinndeutung in der Auseinandersetzung mit den im Lernumfeld aufgefundenen ,Widerständen': Sie ,erfinden' bzw. ,konstruieren' ein neues Stückchen ihrer Mathematik" (Wildt 1998, S. 50).

Ein problemorientierter Unterricht ist im Prinzip so angelegt, dass die Lernenden sich selbständig mit gegebenen Inhalten auseinandersetzen und auf Widerstände reagieren müssen. Die konstruktivistische Sicht des Lernens ist deshalb hierbei in besonderem Maße angebracht. Bei offenen Problemen können die Lernenden darüber hinaus auch die Richtung ihrer Fragestellungen passend ihrer momentanen Vorstellungen mitbestimmen. Die erste Anregung (Pertubation) durch ein innermathematisches oder außermathematisches Problem wird allerdings in der Regel von den Lehrenden ausgehen und dem Entwicklungsstand der Lernenden angepasst werden müssen.

Im Rahmen der konstruktivistischen Sicht des Lernens kann man dann eigentlich auch nicht von „entdeckendem Lernen“ sprechen, da ja eine objektive Welt nicht zugänglich ist; passender wäre der Terminus „erfindendes Lernen“, der zu den Konzeptionen der Behandlung offener Probleme auch besonders gut passt, da hier die Lernenden ja gerade neue Fragestellungen und Zusammenhänge selbst herausfinden sollen. Häufig stoßen sie dabei auch auf Fragestellungen, die selbst für die Lehrenden neu sind und manchmal auch für die Fachwelt neu ist. Jedoch ist Letzteres für das konstruktivistische Lernkonzept nicht wesentlich, es geht immer nur darum, dass es sich aus der Sicht der Lernenden um Erfindungen handelt. Kersten Reich spricht in diesem Zusammenhang auch von „Konstruktion und Rekonstruktion" (vgl. etwa Reich 1997, S. 118-145).

Eine Konsequenz aus der konstruktivistischen Sicht von Lernprozessen im Mathematikunterricht ist es, dass individuelle Vorstellungen über Mathematik einen starken Einfluss auf den Lernprozess haben. Es ist daher wichtig, sich Gedanken zu machen über die verschiedenen Bilder von Mathematik, die Lernende haben könnten. Unter einem Bild von Mathematik (mathematical view) eines Menschen verstehen wir dabei eine Zusammenfassung seiner Vorstellungen, Auffassungen, Sichtweisen, Überzeugungen und Verständnisse über Mathematik einschließlich des Lehren und Lernens von Mathematik (vgl. auch Kapitel 6). Dies funktioniert meistens auf einem unterbewussten Niveau und regelt dabei die Qualität der mathematischen Tätigkeit (z.B. Furinghetti \& Pehkonen 2002). 


$$
\text { "graumann" — 2007/8/21 — 13:22 — page } 261 \text { — \#11 }
$$

$\underline{\text { Problemorientierung im Mathematikunterricht - ein Gesichtspunkt d. Qualitätssteigerung } 261}$

Unser Verständnis über Wissen und Wissenserwerb, bezogen auf Mathematik, war bis ins 20. Jahrhundert geprägt durch den statischen (platonischen) Gesichtspunkt. Das menschliche Wissen ist nach dieser Vorstellung eine wohlstrukturierte Gesamtheit, die man allmählich vermehrt und die in dieser Strukturform angeeignet werden soll. Heute dagegen wird Wissen als dynamischer Prozess, der sich mit der Zeit wandeln kann, angesehen. Deshalb ist das Aneignen der Inhalte nicht das allerwichtigste Ziel, sondern das Lernen der Art und Weise des Datensammelns und der Datenbehandlungsmethoden. Der Prozesscharakter des Wissens wird also betont, entgegen des früher zentralen Produktaspektes (vgl. Freudenthal 1973).

In die gleiche Richtung zielten auch Argumente des ungarischen Mathematikers Imre Lakatos, insbesondere in seinem berühmten Buch „Proofs and Reputations" (Lakatos 1976). Dort äußerte er sich u.a. dahin gehend, dass die platonische und die formalistische Philosophie der Mathematik nicht das Gesamtbild des Gebietes wiedergeben könne, weil sie Mathematik nur statisch beschreibt. Lakatos dagegen versteht Mathematik dynamisch, wobei Beweise (proofs) und Widerlegungen (refutations) miteinander streiten (vgl. etwa auch Mason 1982, Zimmermann 1990). Angeregt von Lakatos stellen P. Davis und R. Hersh sowohl die platonische als auch die formalistische Auffassung von Mathematik in Frage und entwickeln ein Verständnis von Mathematik, das der Erfahrung des aktiv produzierenden Mathematikers entspricht (Davis \& Hersh 1981, Hersh 1997). Dabei ergibt es sich, dass mehrere „richtige“ Bilder von Mathematik nebeneinander existieren. Zum Beispiel benötigte Roberta Mura (1993) vierzehn Kategorien, um die Antworten der kanadischen Mathematiker $(N=104)$ zu der Frage „Was ist Mathematik?" einzuordnen, und dabei war das platonische Bild von Mathematik nur eine Alternative.

Während also in der Vergangenheit eher statische Auffassungen vom Mathematiklernen vorherrschend waren, ist nun die Vorstellung unstrittig geworden, dass Lernen als dynamischer Prozess angesehen werden muss und optimal über die Selbstaktivität der Lernenden erfolgt.

\section{Erläuterungen zu Begriffen aus dem Kontext Problemorientierung}

In der erziehungswissenschaftlichen wie in der mathematikdidaktischen Literatur findet man unterschiedliche Festlegungen und Erklärungen darüber, was unter einem Problem zu verstehen sei.

Das Wort „Problem" stammt aus dem Griechischen und bedeutet soviel wie „das Vorgelegte“, insbesondere auch „die vorgelegte zu lösende Aufgabe“ bzw. „die 
Fragestellung“ oder „die Schwierigkeit“. Die zuletzt genannte Bedeutung tritt uns auch meist in der umgangsprachlichen Bedeutung von „Problem" (z.B. in der Redewendung „Wo liegt das Problem?“" oder „Damit wirst du ein Problem haben!“) entgegen. In einem pädagogischen Lexikon heißt es: „Ein Problem entsteht dann, wenn eine Person nicht in der Lage ist, einen angestrebten Endzustand unmittelbar zu erreichen" (Weis 1971, S. 349). Der bekannte Kreativitätsforscher Dietrich Dörner erklärt ein Problem als einen Zustand, der aus irgendwelchen Gründen für nicht wünschenswert gehalten wird, wobei diejenige Person, die versucht, ein Problem zu lösen, beim Auftreten des Problems nicht über die Mittel verfügt, diesen unerwünschten Zustand in einen erwünschten Zustand zu überführen (Dörner 1987, S. 10). In den USA und einigen anderen Ländern wird der Begriff „Problem“ („problem“) teilweise sehr viel weiter verwendet als hier; z.B. wird mit dem Begriff , word problem“ oft nur eine in Text gekleidete Aufgabe, die nicht unbedingt ein Problem (in unserem Sinne) für die Lernenden darstellt, verstanden.

Wir wollen für unsere Belange in Anschluss an Dörner und nach Kantowski (1980, S. 195) die folgende im deutschsprachigen Raum übliche Definition verwenden:

Ein Problem ist eine für die Löserperson neue Situation, die mit für die Löserperson bekannten Verfahren nicht routinemäßig bewältigt werden kann.

Bei unserer Definition wird deutlich, dass dieses Konzept relational ist, also von dem Individuum und von der Zeit abhängt. Beispielsweise ist für ein Kind der vierten Klasse eine einfache Multiplikationsaufgabe kein Problem, für ein Kind der zweiten Klasse kann es aber eines sein. Die zu findende Lösungsmethode hängt natürlich nicht nur vom Alter, sondern insbesondere auch von den Kenntnissen und Fähigkeiten des Individuums ab.

Ein Problem kann zunächst einmal in drei Aspekte gegliedert werden: die Problemstellung, die Problembearbeitung und die Endsituation.

Bei den klassischen Problemen sind dabei die Problemstellung und die gewünschte Endsituation klar vorgegeben, gesucht ist ein Lösungsweg. Wir sprechen in einem solchen Fall von geschlossenen Problemen.

Liegt ein Problem vor, bei dem entweder die Fragestellung noch gewisse Interpretationen bzw. Variationen zulässt oder/und das gesuchte Ergebnis nicht klar vorgegeben ist bzw. zu weiteren Fragestellungen Anlass gibt, so sprechen wird von einem offenen Problem (open problem). D. h. bei einem offenen Problem ist die Anfangs- oder/und Endsituation des Problems offen (vgl. etwa Nohda 
1991, Pehkonen 1995b, Becker \& Shimada 1997). Hierbei kann man dann wiederum drei verschiedene Fälle unterscheiden, je nachdem ob die Anfangssituation offen ist oder die Endsituation offen ist oder ob beide offen sind (vgl. z.B. Hashimoto 2000, Nohda 2000). Im Fall, dass zu Beginn ein klar definiertes Problem gegeben wird, dieses aber verschiedene weitere Bearbeitungen zulässt, ist es im englischsprachigen Raum üblich vom „open-ended problem “ zu sprechen. Im Fall, dass die Fragestellung noch geklärt werden muss und nur das Untersuchungsthema grob bekannt ist, spricht man von einem „open approach“. Im Fall, dass sowohl die Problemstellung als auch das Ziel nicht klar vorgegeben sind, liegt ein „both-sided open problem “ vor. Stehen dabei die Aktivitäten des Suchens nach Fragen und Informationen sowie nach neuen Erkenntnissen im Vordergrund, so verwendet man vielfach auch den Begriff der „Erkundung“(investigation).

Einen besonderen Fall offener Probleme stellen die sogenannten „FermiProbleme" dar, bei denen keine eindeutigen Antworten erwartet werden können, sondern nur Schätzungen verschiedener Art. Vielmehr geht es bei diesen darum, vorgegebene Sachbestände grob gesehen richtig einzuschätzen bzw. sachlich richtig einzuordnen, z.B. wenn es darum geht, die Anzahl der Atome im Weltall oder alle Möglichkeiten der Kombination von Genen bei einer Kreuzung von Arten oder (wie bei einem von Fermi seinen Studierenden genannten Problem) die Anzahl der Klavierstimmer im Chicago der 1920er Jahre abzuschätzen.

Unter einem Problemfeld (problem field) verstehen wir einen Themenbereich, der mehrere inhaltlich miteinander verbundene Probleme enthält. Häufig steht dabei ein Problem (meist ein open-ended problem) im Mittelpunkt, an das sich weitere Fragestellungen anschließen (vgl. Solvang 1994 und Pehkonen 2001a). Man kann deshalb sagen, dass ein Problemfeld durch eine Gruppe von inhaltlich verbundenen Problemen, die ein gemeinsames Ausgangsproblem („generating problem“) haben, charakterisiert ist. Diese Art des Problemlösens bietet mehr Möglichkeiten, in Richtung verschiedener allgemeiner Ziele zu arbeiten ${ }^{9}$.

Entsprechend unserer Definition des Problems können wir Problemlösen als eine Tätigkeit erklären, die auf das Lösen eines Problems zielt, d. h. als einen „Prozess, bei dem früher erworbenes Wissen in einer neuen und unbekannten Situation verwendet wird" (vgl. NCSM 1989, 471). Der Prozess des Problemlösens kann dabei in verschiedene Phasen eingeteilt werden, wobei Drei-Phasen-, Vier-Phasen-, Fünf-Phasen und Sechs-Phasen-Modelle zu finden sind. In Anlehnung an Polya

9 „I especially favour this way of problem-based education and the work on problem fields because it gives more opportunities for different general objectives, [...] and also can lead to mathematical concepts as well as systematic school mathematics." (Graumann 2002, S. 10). 


$$
\text { "graumann" — 2007/8/21 — 13:22 — page } 264 \text { — \#14 }
$$

(1949) können etwa folgende vier Stufen unterschieden werden: Verstehen des Problems (ggf. mit Präzisierung der Fragestellung), Entwicklung eines Lösungsplanes (ggf. mit Besorgen zusätzlicher Informationen), Erarbeiten der Lösung (ggf. mit Zerlegung in Teilprobleme) und Rückschau (mit Interpretation der Lösung).

Der Begriff Aufgabe (task) wird sowohl in der Literatur als auch in der Umgangssprache ebenfalls in verschiedenen Weisen verwendet. Wir wollen hier das Wort „Aufgabe" nicht in einem speziellen Sinn benutzen, sondern wie meist üblich als Oberbegriff von allen möglichen Aufgabentypen und Problemen. In der Literatur findet man viele Versuche, Aufgaben zu klassifizieren. Unter dem Blickwinkel, ob für die Löserperson ein Problem im obigen Sinne vorliegt oder nicht, unterscheidet man meist zwischen produktiven Aufgaben und reproduktiven Aufgaben (Routineaufgaben).

Für unsere Zwecke ist eine Klassifizierung von Getzels \& al. (1975, S. 102) angebracht, bei der systematisch alle Ja-Nein-Möglichkeiten, die bei den zentralen Variablen (Fragestellung, Lösungsmethode, Lösung) vorkommen, durchgespielt werden. Unterscheidet man zusätzlich noch nach Lehrer und Schüler, so gibt es sechs verschiedene Fälle mit je zwei Alternativen, d. h. insgesamt $2^{6}$ Möglichkeiten. Jedoch ergeben dabei nicht alle Möglichkeiten sinnvolle Aufgabensituationen. In der folgenden Tabelle sind die vier wichtigsten dargestellt.

Tabelle 1. Klassifizierung von Aufgabensituationen nach Getzels \& al. mit vier beispielhaften Fällen (wobei + „bekannt" bzw. „vorgegeben“ und - „unbekannt“ bzw. „nicht vorgegeben“ bedeutet)

\begin{tabular}{|c|c|c|c|c|c|c|}
\hline & \multicolumn{2}{|c|}{ Fragestellung } & Lösungsmethode & \multicolumn{2}{c|}{ Lösung } \\
\hline & Lehrer & Schüler & Lehrer & Schüler & Lehrer & Schüler \\
\hline 1 (Routineaufg.) & + & + & + & + & + & - \\
\hline 2 (klass. Problem) & + & + & + & - & + & - \\
\hline 3 (offenes Probl.) & + & - & + & - & + & - \\
\hline 4 (völlig offene S.) & - & - & - & - & - & - \\
\hline
\end{tabular}

Fall 1 beschreibt die üblichen Schulbuchaufgaben (meist Routineaufgaben), bei denen alles vorgegeben ist und die Schüler die Lösung finden müssen. Fall 2 beschreibt eine klassische schulische Problemaufgabe, bei der die Lehrenden genau wissen, worum es geht. Fall 3 ist ein Fall eines offenen Problems wie es bei Erkundungen in Problemfeldern (vgl. etwa Graumann 1999, 2000 oder 2002) auftritt. Fall 4 ist eine extreme Situation: keiner weiß vorher genau, worum es geht. 
Der Lehrer besorgt den Schülerinnen und Schülern eine Situation - wie z.B. die Spirale von Ulam (vgl. etwa Zimmermann 1986) - und fordert sie auf, verschiedene Probleme in der Situation zu finden, um danach mit ihnen gemeinsam ein Problem zum Lösen auszuwählen. Der Lehrer weiß also nicht vorher, welche Probleme die Schülerinnen und Schüler finden und welche behandelt werden.

\section{Verschiedene Ausprägungen der Behandlung offener Probleme im Unterricht}

An der Erforschung offener Probleme und offener Unterrichtsformen besteht in der Mathematikdidaktik (international gesehen) gegenwärtig ein großes Interesse (vgl. etwa Pehkonen 2001a). Offene Probleme, die in besonderer Weise für das Anstreben allgemeiner Ziele geeignet sind, treten in verschiedenen Ausprägungen auf: Z.B. als offene Sachrechenaufgaben (open word problems), Probleme in realen oder realitätsnahen Situationen (real world problems), Erkundungen (investigations), Projekte (project work), Problemfindungsaktivitäten (problem posing), Aufgabenvariationen und Problemvariationen („what-if“-method) oder als Problemfelder (problem fields / problem sequences / problem domains). Diese Stichwörter geben jeweils nur einen Schwerpunkt an und keine Klassifizierung; Überschneidungen und Verbindungen sind durchaus üblich.

Mathematische Erkundungen (investigations) und die Verwendung von offenen Problemen in Form von open approach werden schon seit etwa 30 Jahren in England und Schottland (vgl. etwa Williams 1989, Morgan 1997) sowie in Australien (vgl. etwa Stacey 1995, McCrae \& Stacey 1997) erprobt. Typisch für diese ist, dass nur eine Ausgangssituation gegeben ist, aus der heraus die Schülerinnen und Schüler das Problem herausarbeiten und lösen müssen. Ein norwegisch-tschechisches Projekt über Problemlösen fokussiert ebenfalls auf die Verwendung offener Probleme (Solvang 1994), wobei in letzter Zeit auch ein besonderes Augenmerk auf Erkundungen (investigations) gelegt wird (Solvang 1997, 1998). Unabhängig von diesem Projekt hat man in der tschechischen Republik unter dem Namen „non-standard problems“ Möglichkeiten und Grenzen der offenen Probleme in Form von investigations erforscht (Trch 1999, Trch \& Zapotilova 1995). Auch in China wird heute sehr viel Gewicht auf die Realisierung offener Probleme im Mathematikunterricht gelegt (vgl. etwa Wong 2000). In Japan ist die Verwendung von offenen Problemen in der Form von „open approach“ gegenwärtig sogar ein fundamentaler Bestandteil der Mathematikdidaktik (vgl. Nohda 1995, Nohda \& Emori 1997, Hashimoto \& Becker 1999, Nohda 2000). 


$$
\text { "graumann" — 2007/8/21 — 13:22 — page 266 — \#16 }
$$

Offene Sachrechenaufgaben (open word problems) im Sinne von Sachrechenaufgaben ohne Fragestellung ${ }^{10}$ bzw. noch zu besorgenden Informationen oder in Form von komplexen Sachrechenproblemen traten schon in der traditionellen Sachrechendidaktik auf. Ende der 1970er Jahre forderte H. Winter den verstärkten Umgang mit „produktiven Sachaufgaben“ (vgl. Winter 1977), die ebenfalls in diese Kategorie zu zählen sind. In neuerer Zeit sind hier vor allem Aufgaben im Rahmen des sogenannten „Neuen Sachrechnens" mit der Forderung nach selbsttätigem Erfinden von Textaufgaben bzw. Rechengeschichten (vgl. etwa Dröge 1995, Schütte 1997) und der mathematischen Durchdringung von kleineren Sacheinheiten (vgl. etwa Erichson o. J., Wittmann \& Müller 1990, 1992) zu nennen. Die Bestrebungen nach mehr Eigenproduktionen der Schülerinnen und Schüler können auch in diesem Kontext gesehen werden (vgl. etwa Selter 1995).

Realitätsbezogene Probleme (real world problems) tauchen im anwendungs- bzw. projektorientierten Mathematikunterricht auf (vgl. etwa Graumann 1976, 1977, 1994b, Becker 1979 oder Tagungsberichte der ICTMA-Kongresse und Aufsätze der ISTRON-Reihe). Ein wichtiger Gesichtspunkt in neuerer Zeit ist dabei die Reflexion über Modellierungsprozesse. In den Niederlanden spielt seit über zwanzig Jahren der Realitätsbezug eine starke Rolle, wobei heute sogar flächendeckend der gesamte Mathematikunterricht in den Niederlanden davon erfasst wird (vgl. etwa Treffers 1991, De Lange 1996, Heuvel-Panhuizen 1996, Dwutsches PISA-Konsortium 2001). In Belgien untersucht eine Gruppe von Forschern um L. Verschaffel Schülerfertigkeiten beim Lösen realitätsbezogener Sachaufgaben (Verschaffel \& al. 1999).

Problemfindung bzw. Probleme erstellen (problem posing) ist seit einiger Zeit ein Forschungsfeld der Arbeitsgruppe um E. Silver in den USA (Silver 1993, 1997; vgl. auch Silver \& Cai 1996), wobei auch Verbindungen mit Taiwan (Leung 1997) und Griechenland (Mamona-Downs 1993) bestehen. In Australien hat sich ebenfalls eine Gruppe mit Problemfindung auseinandergesetzt (Stoyanova \& Ellerton 1997, English 1997). In Deutschland hat B. Zimmermann schon vor etwa zwei Jahrzehnten auf diese Variante des Problemlösens hingewiesen (vgl. Zimmermann 1986).

Seit einiger Zeit beschäftigt sich unter dem Label der Aufgabenvariation eine Arbeitsgruppe unter Leitung von H. Schupp mit diesem Themenkomplex (vgl. Schupp 2002, Hein \& Knichel 1999, Henning \& Leneke 2000). Es geht dabei darum, dass Schülerinnen und Schüler verschiedene Variationsprinzipien an

${ }^{10}$ U.a. damit die Schülerinnen und Schüler sich mit dem Sinn der Aufgabenstellung auseinandersetzen. 
einer vorgegeben Aufgabe kennen lernen und anschließend selbst bei weiteren Aufgaben anwenden können. Allgemeine Ziele sind dabei u.a. die Förderung der Kreativität und des produktiven Umgangs mit Aufgaben; Ziele, die insbesondere auch von H. Winter mit seinen Aufgabenfeldern (vgl. z.B. Winter 1984, 1999) vertreten werden. Mit Aufgabenfeldern, die sich ebenfalls an Winters Zielen orientieren, beschäftigt sich in letzter Zeit auch eine Arbeitsgruppe um R. Bruder in Darmstadt (vgl. Bruder 2002, 2004).

Die Verwendung von offenen Problemen in der Form von Problemfeldern wird seit mehr als fünfzehn Jahren in Finnland untersucht (vgl. Pehkonen 1999, 2001c), wobei auch Untersuchungen zur Realisierung der Behandlung von Problemfeldern in der Schule durchgeführt wurden (vgl. Pehkonen 1986, 1992, 1995a, Pehkonen \& Vaulamo 1999). In Deutschland haben die Bedeutung von Problemfeldern im Mathematikunterricht in den letzten Jahren vor allem G. Graumann, F. Heinrich und B. Zimmermann hervorgehoben. Beispielhafte Problemfelder wurden von Graumann und Heinrich konzipiert und diskutiert (vgl. z.B. Graumann 1994a, 1999, 2000, 2002; Heinrich 1999), Fritzlar untersuchte methodische Vorgehensweisen von Lehramtsstudierenden mit einem Problemfeld (Fritzlar 2004), während E. Pehkonen theoretische Aspekte dazu behandelte (Pehkonen 1998, 2001a, 2001b). B. Zimmermann hat in diesem Zusammenhang insbesondere die kreativen und historischen Aspekte bei der Implementation von Problemen im Mathematikunterricht betont (etwa Zimmermann 1995, 1996, 1999a). Eine Entwicklungsarbeit in ähnlicher Richtung unter dem Namen „Rechenketten“ oder "Aufgabenserien" findet sich bei G. Walther bzw. E. Wittmann (vgl. z.B. Walther 1985a, Wittmann 2001, Wittmann \& Müller 1990, 1992).

\section{Argumente für mehr Problemorientierung im Mathematikunterricht}

Um Missverständnissen vorzubeugen, sei zunächst angemerkt, dass es nicht unsere Absicht ist, den gesamten Mathematikunterricht durch verschiedene Formen von Problemorientierung auszufüllen. Vielfältige Erfahrungen über die Unterrichtspraxis zeigen jedoch, dass der Anteil von problemorientierten Unterrichtsphasen am gesamten Mathematikunterricht immer noch sehr gering ist. Um der Forderung nach mehr Problemorientierung im Mathematikunterricht mehr Gewicht zu verleihen, wollen wir deshalb im Folgenden einige Argumente dafür nennen und erläutern. 


$$
\text { "graumann" — 2007/8/21 — 13:22 — page } 268 \text { — \#18 }
$$

Eine erste Gruppe von Argumenten betrifft die Ziele des Mathematikunterrichts. Wir sehen diese eingebettet in die generelle Aufgabe der Schule, Schülerinnen und Schüler so zu fördern, dass sie unabhängig und selbstständig handeln können, ein kritisches Denken an den Tag legen und vielseitige Fähigkeiten besitzen, um sich im gegenwärtigen und zukünftigen Leben in der Gesellschaft zurecht zu finden. Auf den Mathematikunterricht bezogen geht es dabei insbesondere um den Erwerb von Strukturierungs-, Ordnungs- und Systematisierungsfähigkeiten sowie Modellbildungsfähigkeiten einschließlich der kritischen Reflexion von Grenzen mathematischer Modellierungen. Allgemeine Ziele des Mathematikunterrichts betreffen aber auch die Förderung der Teamfähigkeit und die Fähigkeit zur präzisen, aber dennoch allgemein verständlichen Kommunikation über Sachverhalte, die Entwicklung von Fähigkeiten im Umgang mit Problemen einschließlich der selbsttätigen Informationsaufnahme und des aktiven Umgangs mit Daten (Informationen) und Erkenntnissen. (Vgl. z.B. Graumann 1987, 1993, 1998).

An erster Stelle von allgemeinen Lernzielen, die mit einem problemorientierten Mathematikunterricht besonders gut erreicht werden können, steht natürlich die Fähigkeit des selbständigen und kreativen Arbeitens in gegebenen Situationen und die Problemlösefähigkeit, angefangen von dem Sich-Einlassen auf Schwierigkeiten über die Verwendung heuristischer Strategien bis hin zur Fähigkeit, mögliche Weiterentwicklungen zu erkennen. Aber auch die Fähigkeiten des Klassifizierens, Ordnens, Systematisierens und Strukturierens werden in einem problemorientierten Mathematikunterricht benötigt und trainiert. Ein ebenso wichtiges allgemeines Lernziel, das mit den genannten Fähigkeiten eng zusammenhängt, ist der Erwerb von Fähigkeiten des vernetzten Denkens und des Analogisierens, wobei es um das Herstellen von Zusammenhängen und strukturellen Verwandtschaften geht ${ }^{11}$.

Weitere Fähigkeiten, die im problemorientierten Mathematikunterricht gut angestrebt werden können, sind u.a. die Fähigkeit der systematischen Analyse einer gegebenen Situation bzw. eines speziellen Objektbereiches sowie die Fähigkeit des Erkennens notwendiger Information einschließlich des Findens von Wegen für deren Beschaffung. Nicht unerwähnt bleiben sollten in diesem Zusammenhang auch allgemeine Lernziele, die den individuellen Arbeitsstil der Schülerinnen und Schüler und den Umgang mit komplexen Problemen betreffen. Diesbezügliche

11 ,Within such cognitive skills that could be developed with problem-solving are e.g. flexible thinking, logical thinking, analytic observation, evaluation of information, visualisation, forming analogies and deduction." (Pehkonen 1987, S. 73). 
Fähigkeiten werden sowohl in einem späteren Studium als auch in der Arbeitswelt von heute immer wichtiger ${ }^{12}$. Dazu kommen dann noch die Fähigkeiten der Kooperation und des Arbeitens in einem Team sowie des Kommunizierens über Probleme und Ergebnisse. Diese Fähigkeiten können - wie auch alle anderen nicht nur in einem problemorientierten Mathematikunterricht trainiert werden, aber gerade in einem solchen Unterricht sollte die Teamarbeit sowie das Kommunizieren über bestimmte Sachverhalte und das gegenseitige Argumentieren eine wichtige Rolle spielen. Weiterhin kann sich in einem problemorientierten Mathematikunterricht die Mit- und Selbstbestimmungsfähigkeit der Schülerinnen und Schüler wesentlich besser entfalten als in einem stark lehrerzentrierten Unterricht $^{13}$.

Auch sollte nicht vergessen werden, dass bestimmte Kenntnisse über Mathematik zum Kanon des allgemeinbildenden Wissens unseres Kulturkreises gehört. Zum Verstehen der Alltagswelt gehören z.B. Kenntnisse über Aufbau des dezimalen Stellenwertsystems und das Wissen über das babylonische System, auf das die 60er-Teilungen bei der Uhr und bei den Winkelmaßen zurückgehen. Auch das Problem der Quadratur des Kreises und die Bedeutung einer formalen Algebra für die Lösung von Gleichungen muss als Kulturgut des Abendlandes angesehen werden. Zum tiefergehenden Verständnis der Menschheit gehört auch die Erfahrung, dass Menschen sich immer auch mit Fragen auseinander gesetzt haben, die keine unmittelbare Nützlichkeit brachten. Hierzu gehören sowohl rein spielerische Aktivitäten (vgl. etwa Schach, Tangram oder sogenannte Logeleien) als auch religiöse und philosophische (vgl. etwa die Zahlenmystik bei Pythagoras oder die Rolle der regelmäßigen Körper bei Platon). Ebenso sollte man eine zumindest vage Vorstellung davon haben, welche Rolle axiomatisch begründete Strukturen in der Mathematik spielen und wie solche zunächst rein theoretischen Überlegungen manchmal auch praktische Bedeutung (vgl. etwa die Riemannsche Geometrie für

12 „Der Lernende soll sich eine heuristische Arbeitshaltung zu Eigen machen, die auf Verstehen und Entdecken angelegt ist. ... Denken, das ,wirklich' weiterführt, d. h. ,in unmittelbarer lebendiger Auseinandersetzung mit der Sache (zu) eigene(n) Einsichten' (führt)“ (Glatfeld 1987, S. 19).

13 Es ist kein Zufall, dass Dewey seine Methode mit dem Begriff der Erziehung zur Demokratie in Zusammenhang gebracht hat. In Systemen, die auf Gehorsam gegenüber der Obrigkeit abzielen, ist dagegen eine von den Lehrenden und durch den Stoff streng geführte Unterrichtsmethode passender. Nach Überwindung solcher Systeme ist dann aber in einer Demokratie die Selbstbeherrschung und Selbstorganisation gekoppelt mit Einfühlungsvermögen in andere Menschen (Empathie) von großer Bedeutung. Darüber hinaus ist in einer Demokratie die Kooperationsund Kommunikationsfähigkeit sowie die Fähigkeit der gemeinschaftlichen Bearbeitung von Problemen sehr wichtig. 
"graumann" — 2007/8/21 — 13:22 — page 270 — \#20

die Relativitätstheorie) erlangt haben. Insbesondere die Beschäftigung mit Problemen aus der Geschichte der Mathematik kann zum Erreichen solcher Ziele sehr hilfreich sein.

Eine zweite Gruppe von Argumenten für mehr Problemorientierung im Mathematikunterricht betrifft die Einstellung zur Mathematik bzw. das Bild von Mathematik. Damit bei Schülerinnen und Schülern (und auch in der Öffentlichkeit) kein zu einseitiges Bild von Mathematik entsteht, sollten im Mathematikunterricht verschiedene Aspekte des Umgangs mit Mathematik zum Tragen kommen. Leider ist das bisher kaum der Fall. Wie weidlich aus Unterrichtserfahrungen bekannt ist und wie es auch empirische Untersuchungen gezeigt haben (vgl. etwa die TIMSS-Videostudie), ist der Mathematikunterricht in Deutschland sehr stark durch die Reproduktion fertiger mathematischer Begriffe und Sätze einerseits und die Anwendung von Rechen- bzw. Lösungsalgorithmen auf klar vorstrukturierte Aufgaben andererseits dominiert. Diesem einseitigen Bild von Mathematik kann man nun durch ein verstärktes Arbeiten an innermathematischen Problemen und anwendungsbezogenen Problemkontexten oder durch die Erarbeitung neuer Problemstellungen sehr gut begegnen.

In den Unterrichtsphasen eines problemorientierten Mathematikunterrichts können die Schülerinnen und Schüler auch einen Aspekt des Umgangs mit Mathematik erfahren, der für praktizierende Mathematikerinnen und Mathematiker in der Wissenschaft sowie in der Wirtschaft typisch ist. So ergab z.B. eine Befragung von Personalchefs in Bezug auf die gewünschten Qualifikationen von einzustellenden Diplommathematikerinnen bzw. Diplommathematikern an erster Stelle die Fähigkeit, komplexe Probleme bearbeiten zu können. Untermauert wird dieser Gesichtspunkt durch die Geschichte der Mathematik. Probleme innerhalb der Mathematik oder außermathematische Probleme, die mit Hilfe von Mathematik gelöst wurden, stellten oft Angelpunkte in der Entwicklung von Mathematik dar. Die Geschichte der Mathematik macht auch deutlich, dass Probleme in der mathematischen Forschung in der Regel eingebettet sind in einen größeren inneroder außermathematischen Zusammenhang. Ein problemorientierter Mathematikunterricht, der nicht nur das Lösen einzelner Probleme im Blick hat, sondern (wie es oben in den verschiedenen Ausprägungen beschrieben wurde) etwa auch die Behandlung von Problemfeldern und die Entwicklung neuer Fragestellungen (problem posing) mit einbezieht, ist deshalb für das Bild von Mathematik ein wesentlicher Baustein.

Wie schon oben erwähnt wurde, können in den Unterrichtsphasen eines problemorientierten Mathematikunterrichts die Schülerinnen und Schüler darüber 


$$
\text { "graumann" — 2007/8/21 — 13:22 — page } 271 \text { — \#21 }
$$

$\underline{\text { Problemorientierung im Mathematikunterricht - ein Gesichtspunkt d. Qualitätssteigerung } 271}$

hinaus erfahren, dass Mathematik nicht nur als fertiges Produkt eine Bedeutung hat, sondern auch als Prozess gesehen werden muss, wobei dann auch das konstruktivistische Verständnis von Mathematik zum Tragen kommt.

Ein weiterer Komplex von Argumenten betrifft erkenntnis- bzw. lerntheoretische Aspekte. Das konstruktivistische Verständnis von Mathematik ist nicht nur ein Aspekt des Bildes von Mathematik, sondern auch (wie oben schon ausgeführt wurde) ein Grundprinzip für den Erwerb von Erkenntnissen. Im Sinne des Konstruktivismus ist das Verstehen von erworbenem Wissen nur möglich, wenn die lernende Person sich aktiv mit dem zu erwerbenden Wissen auseinandersetzt und es mit dem bisherigen Wissen verknüpft. Eine selbsttätige Auseinandersetzung mit Mathematik ist von daher essentiell für das Lernen von Mathematik. Darüber hinaus ist aus der Lernpsychologie bekannt, dass Dinge, mit denen man sich selbsttätig auseinander gesetzt hat, besser behalten werden ${ }^{14}$. Die selbsttätige Auseinandersetzung mit Mathematik ist natürlich nicht nur auf einen problemorientierten Mathematikunterricht anzuwenden, die aktive Wissenskonstruktion lässt sich in einem solchen Unterricht aber besonders gut initiieren, denn bei der Beschäftigung mit Problemen bzw. Problemfeldern geht es eben nicht nur um das Aufnehmen von vorgefertigter Mathematik. Weiterhin ist bekannt, dass die selbsttätige Auseinandersetzung mit Problemen zu einer stärkeren Motivation, sich für mathematische Fragen zu engagieren und sich auch weiterhin mit Mathematik auseinander zu setzen, führt.

Ein unterrichtsmethodisches Argument für mehr Problemorientierung im Mathematikunterricht betrifft den Gesichtpunkt der Vertiefung und Übung des erworbenen Wissens. Die Beschäftigung mit Problemen im Mathematikunterricht bietet nämlich vielfältige Möglichkeiten zuvor erworbene Kenntnisse und Fertigkeiten anzuwenden und in einem sinnvollen Zusammenhang zu üben. Unterschiedliche bisher erworbene Kenntnisse und Fertigkeiten werden in der Regel bei der Lösung von Problemen und beim Entwickeln neuer Fragestellungen benötigt. Sie werden dadurch gefestigt und ihre Bedeutung wird in einen größeren Kontext gestellt. Ein weiteres unterrichtsmethodisches Argument betrifft den Aspekt

14 „Das Lernen von Mathematik ist umso wirkungsvoller - sowohl im Hinblick auf handfeste Leistungen, speziell Transferleistungen, als auch im Hinblick auf mögliche schwer fassbare bildende Formung -, je mehr es im Sinne eigener Erfahrungen betrieben wird, je mehr Fortschritt im Wissen, Können und Urteilen der Lernenden auf selbständigen entdeckerischen Unternehmungen beruht" (Winter 1989, S. 1). 
der Differenzierung und der Variation der Unterrichtform. Das Lösen von komplexeren Problemen und insbesondere die Erkundung von bestimmten Problemfeldern erfordert unterschiedliche Kenntnisse, Fertigkeiten und Fähigkeiten. Eine Differenzierung nach Interessen bzw. Leistungsdispositionen kann deshalb ganz selbstverständlich vorgenommen werden. Außerdem bietet es sich oft von der Sache aus an, verschiedene Unterrichtsformen - wie Gruppenarbeit, Partnerarbeit, Einzelarbeit und Plenumsdiskussion - zu verwenden. Eine solche Variation von Unterrichtsformen ist aus pädagogischer Sicht angesagt, damit die Konzentration und aktive Mitarbeit der Schülerinnen und Schüler erhalten werden kann. Erfahrungen mit problemorientierten Unterrichtsphasen haben darüber hinaus gezeigt, dass nicht nur die bisher "guten" Schülerinnen und Schüler besser und mehr lernen, sondern dass vor allem auch sogenannte schwache Schülerinnen und Schüler davon profitieren können. Es ist aber auch von Lernzielen her gut, wenn die Lernenden verschiedene Arbeitsformen kennen gelernt haben.

\section{Beliefs als Einflussfaktor des Lernprozesses und der Unterrichtskultur}

In mehreren Forschungsprojekten der letzten Jahre, die unter dem Aspekt der Implementierung von mehr Problemorientierung in der Schule durchgeführt wurden, bemerkte man, dass die affektive Komponente des Lernens oft einen Trägheitsfaktor oder sogar ein Hindernis bilden kann (vgl. z.B. Pehkonen 1995a). Schon vor zwanzig Jahren bemerkte man (vgl. etwa Schoenfeld 1983, Silver 1985), dass die mathematikbezogenen Beliefs des Lerners seine Problemlöseprozesse stark steuern. Deshalb wurde seit den 1980er Jahre die genauere Erforschung von Beliefs forciert (vgl. Pehkonen 1991).

Unter Beliefs (Glaube, Meinungen, Überzeugungen, Vorstellungen) versteht man subjektive (erfahrungsbasierte) Kenntnisse und Gefühle über ein bestimmtes Objekt. Hierbei ist es nicht wichtig, ob zu dem Objekt fundierte objektive Feststellungen vorhanden sind oder nicht. Auch ist die Stabilität der Beliefs kein wesentliches Merkmal. Einige Beliefs können leicht geändert werden, während andere „steinfest" sind. Die ersten Beliefs beziehen sich üblicherweise auf auBerpersönliche Objekte, während die letzteren meist in enger Verbindung mit der Persönlichkeit des Beliefhalters stehen. Sie sind dann mit tiefer liegenden 
persönlichen oder gesellschaftlichen Werten verbunden, deren Änderung eine starke Verunsicherung erzeugen kann ${ }^{15}$. Die mathematischen Beliefs einer Person bilden eine Struktur (belief system), die für gewöhnlich quasilogisch geordnet ist, d. h. die Person alleine setzt die Regel ihrer Logik (Green 1971). Betrachtet man die umfassende Gesamtheit der bewussten und unbewussten Beliefs einer Person sowie deren Erwartungen oder Hypothesen und deren Kombination, so spricht man auch von dem Bild von Mathematik (mathematical view ${ }^{16}$ der Person.

Eine Möglich wäre es auch, die Beliefs in die Theorie der Attitudes (Einstellungen, Haltungen) einzuordnen (vgl. etwa Meinefeld 1994, Grigutsch 1996). Die Attitudes haben dabei drei Komponenten: kognitive, affektive und handlungsleitende bzw. handlungsintentionale (konative). Beliefs werden dann als kognitive Komponente der Attitudes charakterisiert (vgl. Grigutsch \& al. 1998) ${ }^{17}$.

Verschiedene Auffassungen von Mathematik findet man in der Geschichte der Mathematik zu verschiedenen Zeiten, aber auch zeitlich nebeneinander. Eine Auffassung (bzw. ein Verständnis) von Mathematik wird dabei als Belief mit einer starken Bewusstheit und kognitiven Komponente charakterisiert. In der Mathematikdidaktik wurden in jüngster Zeit hierüber unterschiedliche Analysen erstellt (vgl. auch die Ausführungen bezüglich „individuelle Vorstellungen über Mathematik" in Kapitel 2). Ernest (1991a) z.B. nennt drei Auffassungen: instrumentales, platonisches und konstruktivistisches Verständnis von Mathematik. Ein instrumentales Verständnis von Mathematik liegt nach Ernest vor, wenn Mathematik als Werkzeugkasten betrachtet wird. Beim platonischen Verständnis werden die formalen, strukturalen Aspekte von Mathematik betont und bei der dritten Auffassung steht der Prozess der Entwicklung von Mathematik im Vordergrund. Das konstruktivistische Verständnis von Mathematik passt dabei offensichtlich zu der oben behandelten erkenntnistheoretischen Konzeption des Konstruktivismus, nach der es wichtig ist, dass die Lernenden sich aktiv (nicht nur rezeptiv) mit der vorliegenden Sache bzw. Fragestellung auseinandersetzen, um eine kognitive Struktur elaborieren zu können (etwa Davis \& al. 1990).

Wie schon erwähnt, beschäftigten sich weite Teile der Unterrichtsforschung in der Vergangenheit primär mit der kognitiven Wissensvermittlung. Der affektive Bereich, der insbesondere durch die Metakognition der Lernenden bestimmt wird

\footnotetext{
15 Für eine genauere Begriffsklärung vgl. etwa Furinghetti \& Pehkonen 2002.

16 Eine ausführliche Begriffsanalyse dazu findet sich etwa in Berger 2001.

${ }^{17}$ In vielen Befragungen enthalten die Beliefs aber auch einige affektive Elemente. Deshalb wird diese Charakterisierung heutzutage als unbefriedigend angesehen und kaum noch verwendet.
} 
und die Qualität des Lernens wesentlich beeinflusst, wurde eher sekundär eingestuft. In den letzten beiden Jahrzehnten wurde aber diesem Aspekt des Lehrens und Lernens in vielen Ländern mehr und mehr Aufmerksamkeit gewidmet. Auch in der Mathematikdidaktik überall in der Welt sind in den vergangenen 20 Jahren hierzu eine Reihe von Forschungen betrieben worden, die in Deutschland und in Finnland in den letzten zehn Jahren vor allem im Rahmen des MAVI-Projektes (vgl. Pehkonen \& Törner 1998) stattfanden. Die Thematisierung der Metakognition im Unterricht, d. h. eine angeleitete Reflexion der Schülerinnen und Schüler über ihren Umgang mit Mathematik und ihre Problembearbeitungsstrategien, wird in letzter Zeit in Deutschland von verschiedenen Seiten gefordert.

Die Beliefs der Lernenden gegenüber Mathematik und dem Lernen von Mathematik bilden bei der Auseinandersetzung mit Mathematik und dem Elaborieren der kognitiven Strukturen einen wesentlichen regulierenden Faktor (z.B. Pehkonen \& Törner 1996). Das Bild von Mathematik ist charakteristisch für die Art und Weise, wie ein Individuum mathematikbezogene Situationen erlebt. Deshalb beeinflusst es auch stark sein Handeln beim Umgang mit Mathematik. Versteht z.B. ein Schüler oder eine Schülerin unter Mathematik nur Rechnen im Sinne des rein mechanischen Ausführen von Algorithmen (was ja in der Schule weit verbreitet ist und durch so manchen Unterricht auch noch verstärkt wird), so wird es diesem Schüler bzw. dieser Schülerin in der Regel schwer fallen oder sogar unmöglich sein, nicht-rechnerische Aktivitäten, wie etwa kritisches Prüfen oder Informationen sammeln und ordnen oder neue Fragen stellen, zu tätigen.

Ebenso spielen die Beliefs der Lehrenden gegenüber Mathematik und dem Lernen von Mathematik eine wichtige Rolle für das Lernklima und die Unterrichtskultur. Weil die Lehrperson der allerwichtigste Einflussfaktor in der Klasse ist, sind ihre Beliefs sehr einflussreich in Bezug auf die Einstellungen und den Lernprozess der Lernenden. Lerman (1983) hat z.B. festgestellt, dass die Beliefs der Lehrperson deren Unterricht sehr stark lenken. Im problemorientierten Unterricht sind die Lehrenden nicht mehr nur die Lieferer oder Übermittler von Information, sondern vor allem auch die Leitlinie-Gebenden und Unterstützer der Lernenden sowie Organisatoren und Planer des Lernarrangements. Dazu müssen deren Beliefs aber auch passend sein und sich möglicherweise ändern. So können durch nicht passende Beliefs der Lehrenden, insbesondere bei unerfahrenen Lehrenden, sogar positive Aktivitäten von Schülerinnen und Schüler im offenen Unterricht gestört werden (vgl. Blumenfekd \& al. 1991). Umgekehrt führt die Vorstellung von offenem Unterricht als völlig unstrukturierten offenen Unterricht, in dem 
man die Schülerinnen und Schüler ganz allein lässt (was auch teilweise vorzufinden ist) ebenfalls zu keinem befriedigenden Ergebnis. „Offener Unterricht läuft dann Gefahr zum bloßen Aktionismus oder Leerlauf zu führen. [...] Eine klare Strukturierung des schulischen Geschehens ist in der Konzeption des Offenen Unterrichts unerläßlich und kein Widerspruch zur Offenheit. Klare Strukturen, ein erkennbarer ,roter Faden', ob von den Lehrenden und/oder den Schülerinnen und Schülern selbst entwickelt, machen Unterricht erst effizient und für alle Kinder nachvollziehbar" (Jaumann-Graumann 2000, S. 37/38). Ausartungen offenen Unterrichts sind dabei meist auch Ausdruck von traditionellen Vorstellungen, nur in ihr Gegenteil gewandt und oberflächlich mit pädagogischen Trends gekoppelt.

\section{Möglichkeiten und Barrieren bei der Veränderung des Mathematikunterrichts}

Ein erster Schritt zur gewünschten Veränderung der Unterrichtskultur ist die Veränderung der Beliefs der Lehrenden. Zunächst ist einmal klar, dass alle Lehrenden sich während ihrer Tätigkeit an der Schule ändern, wobei wir hier das Ändern ihrer Beliefs und Auffassungen in bezug auf Lehren und Lernen im Auge haben; in der Literatur verwendet man dabei den Term Teacher Change (Änderung des Lehrers bzw. der Lehrerin). Dieser Entwicklungsprozess scheint von außen gesehen (aber auch oft aus der Sicht der Lehrperson selbst) gleichmäßig wachsend zu verlaufen. In Wirklichkeit variiert aber die Wachstumsgeschwindigkeit von Lehrer zu Lehrer und ist von der Zeit abhängig (vgl. etwa Fennema \& Franke 1992). Genauere Erkenntnisse darüber gibt es aber leider noch nicht.

Wie können wir aber nun die Lehrerinnen und Lehrer dazu bringen, dass sie ihren Unterricht ändern? Wie können wir ihnen helfen, ihr Bild von Mathematik zu verändern? Die Forschung in der Mathematikdidaktik bezüglich der Rolle der Lehrer-Beliefs (vgl. etwa Thompson 1992) hat gezeigt, dass diese sich ändern können, aber man war nicht in der Lage zu zeigen, wie man sie ändern könnte. Die Lehrerauffassungen über „guten Mathematikunterricht" sind so tief in der Persönlichkeit verwurzelt, dass angeregte Änderungen oft auf der Oberfläche blieben. Ändern der äußeren Bedingungen (u.a. Curriculum, Unterrichtsmaterialien) haben keinen grundlegenden Einfluss auf die Beliefs und die Unterrichtskultur gehabt. Falls ein Lehrer bzw. eine Lehrerin gezwungen wird, seinen bzw. ihren Unterricht zu ändern, versucht er bzw. sie in der Regel sich dadurch anzupassen, dass z.B. der Unterricht auf eine neue Weise interpretiert wird und einige 


$$
\text { "graumann" — 2007/8/21 — 13:22 — page 276 — \#26 }
$$

neue Ideen in den alten Unterrichtsstil integriert werden. In der Tat scheint es eine große Kluft zwischen den Beliefs von Lehrenden und ihren Unterrichtsrealisationen zu geben (vgl. z.B. Jones \& al. 1986). Beispielsweise kann ein Lehrer behaupten, dass das Untersuchen der mathematischen Situationen wichtiger als Routineübungen ist, er kann aber gleichzeitig seinen Schülern reichlich mechanische Übungsaufgaben in den Mathematikstunden geben (vgl. z.B. Shaw 1989). Ein anderer Lehrer kann glauben, dass er durch Schülerideen die Klassendiskussion steuert, in der Wirklichkeit akzeptiert er aber nur solche Ideen, die zu seiner Unterrichtsplanung passen (vgl. z.B. Perkkilä 2003).

Das Gemeinsame der bisherigen Forschungsresultate scheint die Beobachtung zu sein, dass die Änderung ein sehr langsamer Prozess ist und dass man noch keine Methode für dessen Beschleunigung gefunden hat. Durch Forschung verstehen wir den Änderungsmechanismus besser als früher, aber die Hauptfrage (Wie könnte man die Änderung beschleunigen?) ist immer noch ungelöst in dem Sinne, dass durch Forschung bisher noch keine Rezepte zur Änderungen gefunden wurde. Sehr oft kann man in den Forschungsberichten lesen, dass bei durchgeführten Interventionen einige Lehrer sich geändert haben und einige nicht (z.B. Senger 1999, Borko \& al. 2000, Wood 2001, Hart 2002). Und ähnliche widersprüchliche Resultate werden immer noch berichtet. Der Einfluss der Intervention scheint oft auch nur solange zu dauern, wie die Forscher in den Klassenzimmern arbeiten (z.B. Cobb \& al., 1990). Danach erfolgt eine Rückwendung („folding back“) zu den alten Verhaltensweisen und die Lehrer-Beliefs scheinen sich nicht geändert zu haben. Eine entsprechende Beobachtung hatte man schon zwanzig Jahre vorher dokumentiert: In der Forschung von Tom Cooney (1985) wurde ein Lehrerstudent (Fred) während seines letzten Studienjahres und ersten Schuljahres bezüglich seiner Beliefs interviewt und sein Unterricht in der Schule beobachtet. Innerhalb des ersten Lehrerjahres konnte man sehen, dass er in der Schulpraxis zu seinen früheren Beliefs über Mathematikunterricht zurückkehrte.

Ein paar Hinweise kann man dennoch finden, die wir hier als Ideen der Bewältigung der Änderungsproblematik präsentieren möchten. Die Erste ist das Anwenden der Theorien der begrifflichen Änderung (conceptual change), mit der die Schwierigkeit der Änderung erklärt wird (z.B. Merenluoto 2001). Diese Forschungsrichtung stammt aus der Didaktik der Naturwissenschaften, zunächst aus den physikalischen Weltbilderklärungen der Kinder (z.B. Vosniadou 1994). In den letzten Jahren hat man angefangen, diese Gedanken auch im Mathematikunterricht anzuwenden (z.B. Lehtinen \& al. 1997). Die Grundidee in diesen Theorien ist, die begrifflichen Änderungen in zwei Gruppen zu zerlegen: altes 
Wissen mit neuem zu bereichern (enrichment) und eine strukturelle begriffliche Änderung (new structuring) - die letztere wird auch oft eine radikale begriffliche Änderung genannt. Bei ersterem kann neues Wissen zu der alten Wissensstruktur hinzugefügt werden, dagegen in den letzteren muss zusätzlich eine Änderung in den Auffassungen des alten Wissens stattfinden. Und eben dies macht die radikale Änderung schwierig. In der Lehreränderungsproblematik kann man denken, dass die benötigte Änderung („vom Wissensvermittler zum Schülerberater“) eine radikale begriffliche Änderung ist. Die Änderung erfüllt die vorgegebenen Bedingungen: Neues Wissen wird zur pädagogischen Wissensstruktur der/des Lehrenden hinzugefügt, gleichzeitig muss aber auch die Auffassung über Lehren geändert werden. Solches scheint vielen Lehrenden eine zu große Änderung zu sein, die nicht leicht zu akzeptieren ist.

Ein Zweites ist eine Änderung in der Fragestellung. Leila Pehkonen (2001, 2004) schlägt vor, dass wir die Frage umkehren sollten. Anstatt Möglichkeiten der Änderung des Lehrers bzw. der Lehrerin sollten wir die konstanten Faktoren des Schulunterrichts untersuchen. Eines ihrer Forschungsprobleme war folgendes: „Welche Faktoren im Lehren und Lernen der Schulmathematik bilden gute und feste Elemente nach Meinungen der Lehrenden?" Sie interviewte neun Primarstufenlehrer und -lehrerinnen und fragte sie u.a. „Was wäre das Letzte, das Sie in der Schule ändern würden?" Mit Hilfe der Themeninterviews folgerte sie, dass hinter den Lehrerantworten deren Bestreben steckte, eine respektierte Lehrerperson zu sein. Man sieht leicht ein, dass ein Lehrer bzw. eine Lehrerin von Kollegen und Eltern respektiert werden möchte. Falls dies eine stichhaltige Schlussfolgerung ist, dann wird es einen neuen Einstieg zur Realisierung der Lehreränderung eröffnen, wenn wir es schaffen, die Werte der Lehrenden in dem Sinne zu ändern, dass eine respektierte Lehrperson in erster Linie eine innovative ist.

\section{Schlussbemerkung}

Unser Hauptanliegen ist die Förderung von Problemorientierung in der Art und Weise, dass sowohl Lehrende als auch Schüler und Schülerinnen die Beschäftigung mit Mathematik sinnvoll erleben und in gewissem Maße auch genießen können. Dabei müssen kognitive Ziele und affektive Ziele im Auge behalten werden. Gemäß der heutigen Auffassung über Unterricht sollte man auch die mathematische Kommunikation in der Klasse vermehren; d. h. Diskussionen zwischen den Lehrenden und den Lernenden sowie zwischen den Schülerinnen und Schülern sollten gefördert werden und auf das Zuhören von Diskussionspartnern muss mehr 
Gewicht gelegt werden (vgl. etwa Pehkonen \& Ejersby 2004). Nur so bietet sich eine Möglichkeit, die Wissenskonstruktion im Sinne der konstruktivistischen Sichtweise zu entwickeln. Folgerungen für den Unterricht sind u.a.: Mehr Spielraum und Zeit für die Selbsttätigkeit der Schülerinnen und Schüler, geeignete Probleme mit der Möglichkeit der Ausweitung auf weiterführende Fragestellungen, realitätsbezogene Probleme mit erkennbarer Relevanz für das Alltagsleben, etc. Außerdem sollten die Lehrenden den Lernenden wirklich zuhören und mögliche „falsche“ Antworten für erneute Reflexionen nutzen.

Von den Lehrenden ist u.a. zu fordern, dass sie sich Kenntnisse über verschiedenartige Probleme bzw. Problemfelder besorgen, den Umgang mit offenen Situationen lernen und dabei ihre Selbstsicherheit nicht verlieren sowie Widersprüche ertragen und widersprüchliche Situationen akzeptieren können. Auch sollte die Öffnung des Klassenzimmers (im Sinne der Zusammenarbeit und gegenseitigen Beobachtung, nicht der Überprüfung und der Durchführung von formellen Vergleichtests) zur Selbstverständlichkeit werden.

Ein Schlüsselkonzept könnte in „mehr Flexibilität“ liegen. Starren Auffassungen in der einen oder anderen Form von Lernenden und Lehrenden bezüglich der Realisierung des Unterrichts begegnet man immer wieder, da die tradierten Einstellungen sich nicht von selbst verändern (oder das Verändern der Einstellungen zu langsam passiert) und eine Tendenz zum Beharren in sich tragen. Veränderungen des Mathematikunterrichts bedürfen deshalb auch immer Veränderungen der Beliefs über Mathematik und das Lernen von Mathematik. Wie in wirkungsvoller Weise eine Veränderung der Beliefs z.B. bezüglich Problemorientierung im Mathematikunterricht erfolgen kann, ist bislang noch unbekannt. Forschungen hierzu, die im Zusammenhang mit Unterrichtserprobungen stehen, sind deshalb unbedingt notwendig.

\section{Literatur}

[1] D. P. Ausubel, Learning by discovery: Rationale and mystique, Bulletin of National Association of Secondary School Principals 45 (1961), 18-58.

[2] D. P. Ausubel, J. D. Novak and H. Hanesian, Educational Psychology: a cognitive view, Holt, Rinehart \& Winston, New York, 1968, (Dt.: Psychologie des Unterrichts, Beltz, Weinheim, 1974).

[3] G. Becker, Anwendungsorientierter Mathematikunterricht in der Sekundarstufe I, Klinkhardt, Bad Heilbrunn, 1979.

[4] J. Becker and L. Shimada, The open-ended approach, NCTM, Reston (VA), 1997. 
[5] P. Berger, Computer und Weltbild. - Habitualisierte Konzeptionen von der Welt der Computer, Westdeutscher Verlag, Wiesbaden, 2001.

[6] D. E. Berlyne, Neugier und Erziehung, in: Entdeckendes Lernen, (H. Neber, Hrsg.), Beltz, Weinheim und Basel, 1973, (Engl. Original 1965).

[7] P. C. Blumenfeld, E. Soloway, R. W. Marx, J. S. Krajcik, M. Guzdial and A. Palincsar, Motivating project-based learning: Substaining the doing, supporting the learning, Educational Psychologist 26, no. 3 \& 4 (1991), 369-398.

[8] H. Borko, K. H. Davinroy, C. L. Bliem and K. B. Cumbo, Exploring and supporting teacher change: Two third-grade teachers' experiences in a mathematics and literacy staff development project, The Elementary School Journal 100 (2000), 273-306.

[9] T. Botts, Problem Solving in Mathematics, The Mathematics Teacher 58 (1965), 496-500 and 596-600.

[10] British Council \& Nuffield Foundation, Mathematics: From primary to secondary, (A Nuffield / British Council Project), Chambers, Edinburgh, 1978.

[11] R. Bruder, Lernen, geeignete Fragen zu stellen. Heuristik im Mathematikunterricht, Mathematik lehren 115 (2002), 4-8.

[12] R. Bruder, Problemlösen lernen als Teil mathematischer Allgemeinbildung - aber wie?, 2004, in: http://www.math-learning.com/files/bremen230604.pdf.

[13] J. S. Bruner, The process of education, Harvard Univ. Press, Cambridge (1960), (Dt.: Der Prozeß der Erziehung, Schwann, Berlin, 1970).

[14] J. S. Bruner, The act of discovery, Harvard educ. Rev. 31 (1961), 21-32.

[15] J. Cai, Problem-Based Mathematics Instruction: Promises and Challenges - a viewpoint from the States, in: Problem Solving Around the World, (E. Pehkonen, ed.), University of Turku, Faculty of Education, 2001, 23-34, Report Series C:14.

[16] P. Cobb, T. Wood and E. Yackel, Classrooms as Learning Environments for Teachers and Researchers, in: Constructivist Views on the Teaching and Learning of Mathematics, JRME Monograph Number 4, (Davis \& al., eds.), NCTM, Reston (VA), 1990, 125-146.

[17] T. J. Cooney, A Beginning Teacher's View of Problem Solving, Journal for Research in Mathematics Education 16, no. 5 (1985), 324-336.

[18] F. Copei, Der fruchtbare Moment im Bildungsprozeß, Quelle \& Meyer, Heidelberg, 1950.

[19] P. J. Davis and R. Hersh, The Mathematical Experience, Birkhäuser, Basel/ Boston/Stuttgart, 1981, (Dt.: Erfahrung Mathematik, Birkhäuser, Basel/Boston/Stuttgart, 1985).

[20] R. B. Davis, C. A. Maher and N. Noddings (eds.), Constructivist Views on the Teaching and Learning of Mathematics, JRME Monograph Number 4, NCTM, Reston (VA), 1990.

[21] J. de Lange, Real problems with real world mathematics, in: Proceedings of the $8^{\text {th }}$ International Congress on Mathematical Education, Sevilla, July 1996, (C. Alsina, 
J. M. Alvarez, M. Niss, A. Pérez, L. Rico and A. Sfard, eds.), S. A. E. M. Thales, Sevilla, 1996, 83-110.

[22] F. Denk, Bedeutung des Mathematikunterrichts für die heuristische Erziehung, Der Mathematikunterricht 10, no. 1 (1964), 36-57.

[23] Deutsches PISA-Konsortium (Hrsg.), PISA 2000. Basiskompetenzen von Schülerinnen und Schülern im internationalen Vergleich, Leske + Budrich, Opladen, 2001.

[24] J. Dewey, Demokratie und Erziehung, Westermann 1949, Braunschweig, 1949, (Engl. Original 1915).

[25] D. Dörner, Problemlösen als Informationsverarbeitung, Kohlhammer, StuttgartBerlin-Köln-Mainz, 1987, 3. Aufl.

[26] R. Dröge, Kinder schreiben Sachaufgaben selbst - Sachrechnen an Situationen orientiert, Die Grundschulzeitschrift, Sonderdruck Mathematik, Bd 2: Geometrie und Sachrechnen (1995), 63-64.

[27] K. Duncker, Zur Psychologie des produktiven Denkens, Springer, Berlin, 1935, (Neudruck 1974).

[28] L. English, Development of Seventh-Grade Students' Problem Posing, in: Proceedings of the PME-21 at Lahti, Vol. 2, (E. Pehkonen, ed.), Lahti Research and training center, University of Helsinki, 1997, 241-248.

[29] C. o. J. Erichson, Von Lichtjahren, Pyramiden und einem regen Wurm - Erstaunliche Geschichten, mit denen man rechnen muß, Verlag für pädagogische Medien, Hamburg.

[30] P. Ernest, The Philosophy of Mathematics Education, The Falmer Press, Hampshire (U.K.), 1991a.

[31] P. Ernest, Constructivism. The Psychology of Learning, and the Nature of Mathematics: Some critical issues, in: Psychology of Mathematics Education (PME) XV, Assisi, 1991b, 25-32.

[32] P. Ernest, Varieties of constructivism: Their metaphors, epistemologies and pedagogical implications, Hiroshima journal of mathematics education, 2/1994 (1994), $1-14$.

[33] E. Fennema and M. L. Franke, Teachers' knowledge and its impact, in: Handbook of research on mathematics learning and teaching, (D. A. Grows, ed.), Macmillan, New York, 1992, 147-164.

[34] J. Foster, Aktives Lernen - Konzeption des entdeckenden Lernens im Primarbereich, Otto Maier Verlag, Ravensburg, 1974, (Engl. Original mit dem Titel „Discovery Learning in the Primary School“, 1972, zweite deutsche, veränderte und aktualisierte Auflage (G. Neff, Hrsg.), Ehrenwith, München, 1993).

[35] H. Freudenthal, Mathematik als pädagogische Aufgabe, Bd. 1, Klett, Stuttgart, 1973.

[36] T. Fritzlar, Zur Sensibilität von Studierenden für die Komplexität problemorientierten Mathematikunterrichts, Kovac, Hamburg, 2004. 
[37] L. Führer, Dreihundert Jahre Theorie des öffentlichen Mathematikunterrichts in Deutschland, in: Beiträge zum Mathematikunterricht 2000, Franzbecker, Hildesheim, 2000, 19-26.

[38] F. Furinghetti, A theoretical framework for teachers' conceptions, in: Current State of Research on Mathematical Beliefs III. Proceedings of the MAVI-3 Workshop, (E. Pehkonen, ed.), University of Helsinki, Department of Teacher Education, 1996, 19-25, Research Report 170.

[39] F. Furinghetti and E. Pehkonen, Rethinking Characterizations of Belief, in: Beliefs: A hidden variable in mathematics education?, (G. Leder, E. Pehkonen and G. Törner, eds.), Kluwer, Dordrecht, 2002, 39-57.

[40] H. Gaudig, Die Schule im Dienste der werdenden Persönlichkeit, 3. Auflage, Quelle \& Meyer, Leipzig, 1930.

[41] J. W. Getzels and M. Csikszentmihalyi, From Problem Solving to Problem Finding, in: Perspectives in Creativity, (I. A. Taylor and J. W. Getzels, eds.), Aldine Publishing Company, Chicago, 1975, 90-116.

[42] E. v. Glasersfeld (ed.), Radical constructivism in mathematics education, Kluwer, Dordrecht, 1991.

[43] E. v. Glasersfeld, Aspekte einer konstruktivistischen Didaktik, LSW (1995), 7-14.

[44] E. v. Glasersfeld, Radikaler Konstruktivismus: Ideen, Ergebnisse, Probleme, Suhrkamp, Frankfurt/M, 1996.

[45] M. Glatfeld (Hrsg.), Mathematik lernen - Probleme und Möglichkeiten, Vieweg, Braunschweig, 1977.

[46] M. Glatfeld, Überlegungen zum Induktionsbegriff - unter fachdidaktischer Hinsicht, Europäische Hochschulschriften Band 287, Frankfurt/M-Bern-New York-Paris, 1987.

[47] G. Graumann, Praxisorientiertes Sachrechnen, in: Beiträge zum Mathematikunterricht 1976, Schroedel, Hannover, 1976, 79-83.

[48] G. Graumann, Praxisorientierter Geometrieunterricht, in: Beiträge zum Mathematikunterricht 197r, Schroedel, Hannover, 1977, 98-101.

[49] G. Graumann, Geometry in Everyday Life, in: Articles on Mathematics Education, (E. Pehkonen, ed.), University of Helsinki, Department of Teacher Education, 1987, 11-23, Research Report 55, [Vgl. auch Geometrie im Alltag, G. Graumann, in: Mathematik lehren Heft 29 / 1988, 1988, 8-14].

[50] G. Graumann, Die Rolle des Mathematikunterrichts im Bildungsauftrag der Schule, Pädagogische Welt, Heft 5/1993 (1993), 194-199 (und 204).

[51] G. Graumann, Entdeckendes Lernen im Geometrieunterricht, in: Beiträge zum Lernen und Lehren von Mathematik, (F. Padberg, Hrsg.), Kallmeyersche Verlagsbuchhandlung, Seelze (Velber), 1994a, 60-74.

[52] G. Graumann, Geometrie im Alltag, in: Materialien für einen realitätsbezogenen Mathematikunterricht (ISTRON-Reihe Band 1), (W. Blum, Hrsg.), Franzbecker, Bad Salzdetfurth, 1994b, 31-59. 
[53] G. Graumann, Ordnen, Strukturieren, Systematisieren - Gewinn jenseits des Rechnens, in: Mathe, ja bitte - Wege zu einem anderen Unterricht, (G. Graumann und K. Röttel, Hrsg.), Polygon Verlag, Buxheim-Eichstätt, 1998, 47-53.

[54] G. Graumann, Entdeckungen in Problemfeldern mit Beispielen aus der Dreieckslehre der SI, in: Beiträge zum Mathematikunterricht 1999, Franzbecker, Hildesheim, 1999.

[55] G. Graumann, Regelmäßige Vielecke - ein Problemfeld für Systematisierungsübungen, in: Beiträge zum Mathematikunterricht 2000, Franzbecker, Hildesheim, 2000.

[56] G. Graumann, Problem Orientation in Geometry Teaching, in: Tutkimuksella parempaan opetukseen. Report from the Department of Teacher Education in Tampere University, A 26/2002, (H. Silfverberg and J. Joutsenlahti, eds.), 2002, 9-23.

[57] G. Graumann, R. Hölzl, K. Krainer, M. Neubrand und H. Struve, Tendenzen der Geometriedidaktik der letzten 20 Jahre, Journal für Mathematik-Didaktik 17, no. 3/4 (1996), 163-273.

[58] T. F. Green, The Activities of Teaching, McGraw-Hill Kogakusha, Tokyo, 1971.

[59] S. Grigutsch, Mathematische Weltbilder von Schülern. Struktur, Entwicklung, Einflußfaktoren, Universität Duisburg, Fachbereich Mathematik, Duisburg, 1996, Dissertation, (Erweiterte Kurzfassung: Vgl. Journal für Mathematikdidaktik Heft $1 / 98,3-45)$.

[60] S. Grigutsch, U. Raatz und G. Törner, Einstellungen gegenüber Mathematik bei Mathematiklehrern, Journal für Mathematik-Didaktik 19, no. 1 (1998), 3-45.

[61] J. Hadamard, The Psychology of Invention in the Mathematical Field, Dover Publications, New York, 1954, (Orig. 1945).

[62] L. C. Hart, A four year follow-up study of teacher's beliefs after participating in a teacher enhancement project, in: Beliefs: A hidden variable in mathematics education?, (G. Leder, E. Pehkonen and G. Törner, eds.), Kluwer, Dordrecht, 2002, $161-176$.

[63] Y. Hashimoto and J. Becker, The open approach to teaching mathematics - creating a culture of mathematics in the classroom: Japan, in: Developing mathematically promising students, (L. J. Sheffield, ed.), NCTM, Reston (VA), 1999, 101-119.

[64] Y. Hashimoto, Eliciting Mathematical Ideas from Students: Towards its Realization in Japanese Curriculum, in: Abstracts of Plenary Lectures and Regular Lectures, ICME-9, Tokyo/Makuhari, Japan, 2000, 54-55.

[65] W. Hein und H. Knichel, Schüler variieren eine Aufgabe, Mathematische Unterrichtspraxis 20, no. 2 (1999), 33-38.

[66] F. Heinrich, Warum gelang keine Lösung des Problems?, in: Beiträge zum Mathematikunterricht 1999, Franzbecker, Hildesheim, 1999.

[67] H. Henning und B. Leneke, Aufgabenvariationen als Unterrichtsgegenstand, Technical Report Nr. 1 / 2000, Otto-von-Guericke-Universität, Magdeburg, 2000.

[68] H. v. Hentig, Schule als Erfahrungsraum, Klett, Stuttgart, 1973.

[69] R. Hersh, What is mathematics, really?, Jonathan Cape, London, 1997. 
[70] M. Heuvel-Panhuizen, Assessment and realistic mathematics education, Utrecht University, Utrecht, 1996, Dissertation.

[71] C. C. Hill, Problem solving: Learning and Teaching. An annotated bibliography, Nichols, New York, 1979.

[72] H. Hinkfuß, Heuristische Methoden im Mathematikunterricht, Pfeffer, Bielefeld, 1980.

[73] G. Holland, Geometrie in der Sekundarstufe - Didaktische und methodische Fragen, 2. Auflage, Spektrum, Heidelberg-Berlin-Oxford, 1996, (1. Auflage 1988).

[74] G. Howson, Changes in mathematics education since the late 50's. Ideas and realisation, Educational Studies of Mathematics 9, no. 2 (1978), 183-223.

[75] K.-H. Hürten, Das heuristische Prinzip der Geometrie und seine Bedeutung für die Propädeutik des Mathematikunterrichts, in: Beiträge zum Mathematikunterricht 1970, Schroedel, Hannover, 1971, 63-72.

[76] K.-H. Hürten, Das heuristische Prinzip in der Geometrie, Der Mathematisch-Naturwissenschaftliche Unterricht, Heft 5/86 (1976), 276-281.

[77] S. Isaacs, The Children We Teach, University of London Press, London, 1932.

[78] S. Isaacs, Social Development in Young Children, Routledge \& Kegan Paul, London, 1948.

[79] Istron, Materialien für einen realitätsbezogenen Mathematikunterricht, Band 1ff, Franzbecker, Hildesheim, 1994 ff.

[80] O. Jaumann-Graumann, Offener Unterricht - ja, aber strukturiert, Grundschule, 9/2000 (2000), 36-38.

[81] H. C. Johnson, Problem solving in arithmetic: a review of the literature, Elementary School Journal 44 (1944), 396-403 and 476-482.

[82] D. Jones, E. Henderson and T. Cooney, Mathematics teachers' beliefs about mathematics and about teaching mathematics, in: Proceedings of PME-NA 8, (G. Lappan and R. Even, eds.), Michigan State University, East Lansing (MI), 1986, 274-279.

[83] M. G. Kantowski, Some Thoughts on Teaching for Problem-Solving, in: Problem Solving in School Mathematics, NCTM Yearbook 1980, (S. Krulik and R. E. Reys, eds.), NCTM, Reston (VA), 1980, 195-203.

[84] K. Kiesswetter, Kreativität in der Mathematik und im Mathematikunterricht, in: Mathematik lernen, (M. Glatfeld, Hrsg.), Vieweg, Braunschweig, 1977, 1-39.

[85] K. Kiesswetter, Modellierung von Problemlöseprozessen, Der Mathematikunterricht 29, no. 3 (1983), 71-101.

[86] J. Kilpatrick, Problem-Solving and Creative Behavior in Mathematics, in: Review of Research in Mathematics Education, (J. W. Wilson and L. R. Carry, eds.), School Mathematics Study Group, Stanford, 1969a.

[87] J. Kilpatrick, Problem solving in mathematics, Review of Educational Research 39, no. 4 (1969b), 523-534. 
[88] J. Kilpatrick, What Constructivism Might Be in Mathematics Education, in: Psychology of Mathematics Education (PME) XI, Vol. I, (Bergerson et al., ed.), Montreal, 1987, 3-27.

[89] E. Klieme, Mathematisches Problemlösen als Testleitung, Lang, Frankfurt/M, 1989.

[90] W. Köhler, The mentality of apes, New York, 1925.

[91] S. Krulik and R. E. Reys (eds.), Problem Solving in School Mathematics, NCTM Yearbook, NCTM, Reston (VA), 1980.

[92] I. Lakatos, Proofs and Refutations - the Logic of Mathematical Discovery, Cambridge University Press, Cambridge, 1976, (Dt.: Beweise und Widerlegungen: die Logik mathematischer Entdeckungen, Vieweg, Braunschweig, 1979).

[93] E. Lehtinen, K. Merenluoto and E. Kasanen, Conceptual change in mathematics: From rational to (un)real numbers, European Journal of Psychology of Education XII, no. 2 (1997), 131-145.

[94] H. Lenné, Analyse der Mathematikdidaktik in der Bundesrepublik Deutschland, Klett, Stuttgart, 1969.

[95] S. Lerman, Problem-solving or knowledge-centred: the influence of philosophy on mathematics teaching, International Journal for Mathematical Education in Science and Technology 14, no. 1 (1983), 59-66.

[96] S. S. Leung, On the open-ended nature in mathematical problem posing, in: Use of open-ended problems in mathematics classroom, (E. Pehkonen, ed.), University of Helsinki, Department of Teacher Education, 1997, 26-33, Research Report 176.

[97] J. D. Lockard, The Ninth International Clearinghouse on Science and Mathematics Curriculum Development and The Tenth International Clearinghouse on Sc. And Math. Curr. Dev., The Comm. Of Sc. Ed. Of the Am. Ass. For the Advancement of Sc. \& The Sc. Teaching Center of the Univ. of Maryland, Univ. of Maryland, $1975 / 1977$.

[98] J. Mamona-Downs, On analysing problem posing, in: Proceedings of the seventeenth PME conference, Vol. I, (I. Hirabayashi, N. Nohda, K. Shigematsu and F.-L. Lin, eds.), University of Tsukuba, Tsukuba (Japan), 1993, 41-56.

[99] J. Mason (with L. Burton and K. Stacey), Thinking Mathematically, Addison-Wesley, Bristol, 1982.

[100] T. R. McConnel, Discovery vs. authoritarian identification in the learning of children, Stud. Educ. 9 No 5, Univ. of Iowa, 1934.

[101] B. McCrae and K. Stacey, Testing problem solving in a high-stakes environment, in: Use of open-ended problems in mathematics classroom, (E. Pehkonen, ed.), University of Helsinki, Department of Teacher Education, 1997, 34-48, Research Report 176.

[102] W. Meinefeld, Einstellung, in: Handwörterbuch Psychologie, (R. Asander und G. Wenninger, Hrsg.), Beltz, Weinheim, 1994, 120-126. 
[103] K. Merenluoto, Lukiolaisen reaaliluku. Lukualueen laajentaminen käsitteellisenä muutoksena matematiikassa, [Students' real numbers. Enlarging the number concept as a conceptual change in mathematic], Turun yliopiston julkaisusarja, sarja C osa 176, Turun yliopisto, 2001.

[104] C. Morgan, The institutionalisation of open-ended investigation: some lessons from the U.K. experience, in: Use of open-ended problems in mathematics classroom, (E. Pehkonen, ed.), University of Helsinki, Department of Teacher Education, 1997, 49-62, Research Report 176.

[105] MUED (Mathematikunterrichtseinheitendatei), MUED-Tagung „Problemorientierter Mathematikunterricht", Rundbrief 4/79, Heinrich-Behnke-Seminar, Münster, 1979.

[106] W. Münzinger, Projektorientierter Mathematikunterricht, Urban \& Schwarzenberg, München-Wien-Baltimore, 1977.

[107] R. Mura, Images of mathematics held by university teachers of mathematics and sciences, Educational Studies in Mathematics 93 25, no. 4 (1993), 375-385.

[108] NCSM, Essential Mathematics for the Twenty-first Century, Math. Teacher 82, no. 6 (1989), 470-474.

[109] H. Neber, Entdeckendes Lernen, Beltz, Weinheim und Basel, 1973.

[110] H. Neber, Lernen, entdeckendes, in: Enzyklopädie Erziehungswissenschaft, Band 4, (G. Otto und W. Schulz, Hrsg.), Klett, Stuttgart, 1995, 512-514.

[111] J. Neubrand und M. Neubrand, Funktionen multipler Lösungsmöglichkeiten: Beispiele aus einer japanischen Mathematikstunde, Arbeiten aus dem Institut für Mathematik und ihre Didaktik der Bildungswissenschaftlichen Hochschule-Universität Flensburg, Heft 13, 1998.

[112] N. Nohda and H. Emori, Communication and negotiation through open-approach method, in: Use of open-ended problems in mathematics classroom, (E. Pehkonen, ed.), University of Helsinki, Department of Teacher Education, 1997, 63-72, Research Report 176.

[113] N. Nohda, Paradigm of the "open-approach" method in mathematics teaching: Focus on mathematical problem solving, Zentralblatt für Didaktik der Mathematik 23, no. 2 (1991), 32-37.

[114] N. Nohda, Teaching and Evaluating Using "Open-Ended Problem" in Classroom, Zentralblatt für Didaktik der Mathematik 27, no. 2 (1995), 57-61.

[115] N. Nohda, Teaching by Open-Approach Method in Japanese Mathematics Classroom, in: Proceedings of the PME-24 Conference, Vol. 1, (T. Nakahara and M. Koyama, eds.), Hiroshima University (Japan), 2000, 39-53.

[116] E. Pehkonen, Über Bedeutung der Knobelaufgaben, in: Beiträge zum Mathematikunterricht 1986, Verlag Franzbecker, Bad Salzdetfurth, 1986, 227-230.

[117] E. Pehkonen, The meaning of problem solving for childrens's development, in: Articles on Mathematics Education, (E. Pehkonen, ed.), University of Helsinki, Department of Teacher Education, 1987, 71-86, Research Report 55. 
[118] E. Pehkonen, Developments in the understanding of problem solving, Zentralblatt für Didaktik der Mathematik 23, no. 2, 1991, 46-50.

[119] E. Pehkonen, Using Problem Fields as a Method of Change, The Mathematics Educator 3, no. 1, 1992, 3-6.

[120] E. Pehkonen, On Teachers' Beliefs and Changing Mathematics Teaching, Journal für Mathematik-Didaktik 15, no. 3/4, 1994, 177-209.

[121] E. Pehkonen, On pupils' reactions to the use of open-ended problems in mathematics, Nordic Studies in Mathematics Education 3, no. 4, 1995a, 43-57.

[122] E. Pehkonen, Introduction: Use of Open-Ended Problems, Zentralblatt für Didaktik der Mathematik 27, no. 2, 1995b, 55-57.

[123] E. Pehkonen, Introduction: The state-of-art in mathematical creativity, Zentralblatt für Didaktik der Mathematik 29, no. 3, 1997a, 31-35.

[124] E. Pehkonen, Introduction to the concept "open-ended problem", in: Use of open-ended problems in mathematics classroom, 7-11., (E. Pehkonen, ed.), University of Helsinki, Department of Teacher Education, 1997b, Research Report 176.

[125] E. Pehkonen, Über offene Aufgaben im schulischen MU, in: Beiträge zum Mathematikunterricht 1998, Franzbecker, Hildesheim, 1998, 495-498.

[126] E. Pehkonen, On the use of open approach to promote an educational change in mathematics, in: Proceedings of International Symposium Elementary Maths Teaching SEMT 99, (M. Hejny \& J. Novotna, eds.), Charles University, Prague, 1999, 26-33.

[127] E. Pehkonen, Introduction: Problem Solving in Mathematics Education, in: Problem Solving Around the World, 7-9., (E. Pehkonen, ed.), University of Turku, Faculty of Education, 2001a, Report Series C:14.

[128] E. Pehkonen, How Do We Understand Problem and Related Concepts?, in: Problem Solving Around the World, 11-20., (E. Pehkonen, ed.), University of Turku, Faculty of Education, 2001b, Report Series C:14.

[129] E. Pehkonen, Offene Probleme: Eine Methode zur Entwicklung des Mathematikunterrichts, Der Mathematikunterricht 47, no. 6, 2001c, 60-72.

[130] E. Pehkonen and G. Törner (eds.), The State-of-Art in Mathematics-Related Belief Research. Results of the MAVI activities, University of Helsinki, Department of Teacher Education, 1998, Research Report 195.

[131] E. Pehkonen and G. Törner, Mathematical Beliefs and Different Aspects of Their Meaning, Zentralblatt für Didaktik der Mathematik (ZDM) 28, no. 4, 1996, 101-108.

[132] E. Pehkonen and J. Vaulamo, Pupils in lower secondary school solving open-ended problems in mathematics, in: Proceedings of the PME-23 conference, Vol. 4, (O. Zaslavsky, ed.), Israel Institute of Technology, Haifa, 1999, 33-40.

[133] E. Pehkonen and L. R. Ejersbo, Levels of a Teacher's Listening when Teaching Open Problems in Mathematics, in: Proceedings of the PME-NA conference, (D. McDougall, ed.), University of Toronto, 2004 (to appear). 
[134] L. Pehkonen, The matter of change - being a respectable teacher, A paper presented in the PME-24 conference July 12-17, 2001 at the University of Utrecht, Freudenthal Institute, 2001.

[135] L. Pehkonen, The magic circle of the textbooks - an option or an obstacle for teacher change, in: Proceedings of the PME28 conference, Vol. 3, (M. J. Høines and A. B. Fuglestad, eds.), Bergen University College, 2004, 513-520.

[136] P. Perkkilä, Primary school teachers' mathematics beliefs and teaching practices, in: Proceedings of the CERME-3 conference, A draft version in the web site: http://www.dm.unipi.it/ didattica/CERME3/draft/proceedings_draft/TG2_draft/, 2003.

[137] G. Polya, How to solve it, Princeton University Press, Princeton, 1945.

[138] G. Polya, Schule des Denkens: vom Lösen mathematischer Probleme, Francke, Bern, 1949.

[139] G. Polya, Mathematik und Plausibles Schließen I, II, Birkhäuser, Basel/Boston/Stuttgart, 1962/63, (Engl. Original: Mathematics and Plausible Reasoning, Princeton Univ. Press, Princeton, 1954).

[140] G. Polya, Mathematical Discovery I, II, Wiley, New York, 1962/65.

[141] G. Polya, Die Heuristik - Versuch einer vernünftigen Zielsetzung, Der Mathematikunterricht, Heft 1/1964, 1964, 5-15.

[142] G. Polya and J. Kilpatrick, The Stanford Mathematics Problem Book with Hints and Solutions, Teachers College Press, New York, 1974.

[143] K. Reich, Systemisch-konstruktivistische Pädagogik - Einführung in Grundlagen einer interaktivistisch-konstruktivistischen Pädagogik, Luchterhand, Neuwied, 1997.

[144] K. Reich, Thesen zur konstruktivistischen Didaktik, Pädagogik, Heft 7-8/98, 1998, 43-46.

[145] H. Rehlich and B. Zimmermann (eds.), ProMath 2003 - Problem Solving in Mathematics Education, Proceedings of an International Symposium in September 2003, Franzbecker, Hildesheim-Berlin, 2004.

[146] A. H. Schoenfeld, Can Heuristics be taught?, in: Cognitive Process Instruction research on teaching thinking skills, (J. Lochhead and J. Clement, eds.), Franklin Institute Press, Philadelphia, 1979.

[147] A. H. Schoenfeld, Problem solving in the mathematics curriculum: a report, recommandations, and an annoted bibliography, Waschington D.C, 1983.

[148] A. H. Schoenfeld, Mathematical Problem Solving, Academic Press, Orlando (Fl.), 1985.

[149] A. H. Schoenfeld, Cognitive Science and Mathematics Education: An Overview, in: Cognitive Science and Mathematics Education, (A. H. Schoenfeld, ed.), Lawrence Erlbaum Associates, Hillsdale (NJ), 1987, 1-31.

[150] T. L. Schroeder and F. K. Lester, Developing Understanding in Mathematics via Problem Solving, in: New Directions for Elementary School Mathematics, NCTM 
1989 Yearbook, (P. R. Trafton and A. P. Shulte, eds.), NCTM, Virginia USA, 1989.

[151] H. Schupp, Demonstrierender oder explorierender Geometrieunterricht?, Mathematik in der Schule 33, no. 11, 1995, 586-593.

[152] H. Schupp, Aufgabenvariation als Heurismus, Projektbericht, Universität des Saarlandes, 1998.

[153] H. Schupp, Thema mit Variationen oder Aufgabenvariationen im Mathematikunterricht, Franzbecker, Hildesheim, 2002.

[154] S. Schütte, Rechengeschichten statt Textaufgaben: Mathematik und Sprache verbinden, Die Grundschulzeitschrift 102, no. 3, 1997, 6-11.

[155] Chr. Selter, Mathematiklernen innerhalb und ausserhalb der Schule, Beiträge zum Mathematikunterricht, Franzbecker, Hildesheim, 1995, 436-439.

[156] E. Senger, Reflective reform in mathematics: The recursive nature of teacher change, Educational Studies in Mathematics 37, 1999, 199-221.

[157] K. L. Shaw, Contrasts of teacher ideal and actual beliefs about mathematics understanding: Three case studies, Doctoral dissertation, University of Georgia, Athens (GA), 1989, unpublished.

[158] H. Siebert, Lernen als Konstruktion von Lebenswelten - Entwurf einer konstruktivistischen Didaktik, Frankfurt/M, 1994.

[159] H. Siebert, Pädagogischer Konstruktivismus, Luchterhand, Neuwied, 1999.

[160] E. A. Silver, Research on Teaching Mathematical Problem Solving: Some Underrepresented Themes and Needed Directions, in: Teaching and Learning Mathematical Problem Solving: Multiple Research Perspectives, (E. Silver, ed.), Lawrence Erlbaum Associates, Hillsdale (NJ), 1985, 247-266.

[161] E. A. Silver, On mathematical problem posing, in: Proceedings of the seventeenth PME conference, Vol. I, (I. Hirabayashi, N. Nohda, K. Shigematsu and F.-L. Lin, eds.), University of Tsukuba, Tsukuba (Japan), 1993, 66-85.

[162] E. A. Silver, The Nature and Use of Open Problems in Mathematics Education: Mathematical and Pedagogical Perspectives, Zentralblatt für Didaktik der Mathematik 27, no. 2, 1995, 67-72.

[163] E. A. Silver, Fostering Creativity through Instruction Rich in Mathematical Problem Solving and Problem Posing, Zentralblatt für Didaktik der Mathematik 29, no. $3,1997,75-80$.

[164] E. A. Silver and J. Cai, An Analysis of Arithmetic Problem Posing by Middle School Students, Journal for Research in Mathematics Education 27, no. 5, 1996, $521-539$.

[165] R. Solvang, Thoughts about the free phase in connection to the use of generator problem, in: Selected Topics From Mathematics Education 3, (R. Solvang, ed.), Center for Teacher Education and In-Service Training, University of Oslo, 1994, $56-67$.

[166] R. Solvang (ed.), Mathematical Investigations in School Mathematics 1, Department of Teacher Education and School Development, University of Oslo, 1997. 
[167] R. Solvang (ed.), Mathematical Investigations in School Mathematics 2, Department of Teacher Education and School Development, University of Oslo, 1998.

[168] K. Stacey, The Challenges of Keeping Open Problem-Solving Open in School Mathematics, Zentralblatt für Didaktik der Mathematik 27, no. 2, 1995, 62-67.

[169] E. Stoyanova and N. F. Ellerton, Problems in Mathematics Classroom, The Australian Mathematics Teacher 53, no. 4, 1997, 8-13.

[170] E. Terhart, Konstruktivismus und Unterricht - Gibt es einen neuen Ansatz in der Allgemeinen Didaktik?, Zeitschrift für Pädagogik, 45. Jg./1999, 1999, 629-647.

[171] A. G. Thompson, Teachers' Beliefs and Conceptions: A Synthesis of the Research, in: Handbook of research on mathematics learning and teaching, (D. A. Grouws, ed.), Macmillan, New York, 1992, 127-146.

[172] G. Törner und E. Pehkonen, Literature on Beliefs, Schriftenreihe des Fachbereichs Mathematik, Preprint Nr. 341, Universität, Duisburg, 1996.

[173] G. Törner, Views of German Mathematics Teachers on Mathematics, in: Developments in Mathematics Education in Germany, Selected Papers from the Annual Conference of Didactics of Mathematics, Regensburg 1996, (E. Cohors-Fresenborg et al., eds.), Franzbecker, Hildesheim, 1998, 116-130.

[174] M. Trch and E. Zapotilova, Non-standard Problems: The Means of Development of Thinking and Geometric Imagination in the Lowest School Age, in: Proceedings of International Symposium Elementary Maths Teaching SEMT 95, (M. Hejny and J. Novotna, eds.), Charles University, Prague, 1995, 62-65.

[175] M. Trch, Use of grids: covering of the plane with congruent polyomino tiles, in: Proceedings of International Symposium Elementary Maths Teaching SEMT 99, (M. Hejny and J. Novotna, eds.), Charles University, Prague, 1999, 111-115.

[176] A. Treffers, Realistic Mathematics Education in the Netherlands 1980-1990, in: Realistic Mathematics Education in primary school, (L. Streefland, ed.), CD-Press, Utrecht, 1991.

[177] Ö. Vancso, Die Begabtenförderung in Ungarn, Beiträge zum Mathematikunterricht 2002, Franzbecker, Hildeheim, 2002, 47-54.

[178] A. L. Veistinen (ed.), Proceedings of The Pro Math Workshop in Turku May 24-27, 2001, University of Turku Department of Techer Education Pre-Print nr. 1, 2002.

[179] L. Verschaffel, E. de Corte, S. Lasure, G. van Vaerenbergh, H. Bogaerts and E. Ratinckx, Learning to solve mathematical application problems: A design experiment with fifth graders, Mathematical Thinking and Learning 1, no. 3, 1999, 195-229.

[180] S. Vosniadou, Capturing and modeling the process of conceptual change, Learning and Instruction 4, 1994, 45-69.

[181] G. Walther, Problemlösen in einer Hauptschulklasse, Pädagogische Welt 10, 1984, $10-12$.

[182] G. Walther, Rechenketten als stufenübergreifendes Thema des Mathematikunterrichts, Mathematik lehren 11 (August 1985a), 16-21. 
[183] G. Walther, Eine einfache Aufgabe - und was man daraus als Lehrer für problemorientierten Unterricht lernen kann, Didaktik der Mathematik 6, 1985b, 295-302.

[184] V. Weis, Stichwort "Problemlösen", in: Lexikon der Pädagogik, Band 3, H. Rombach (Hrsg.), Herder, Freiburg, 1971, 349.

[185] M. Wertheimer, Productive thinking, Harper \& Row, New York, 1945, (Dt.: Produktives Denken, Kramer, Frankfurt/M, 1957).

[186] T. Weth, Kreativer Mathematikunterricht, Franzbecker, Hildesheim, 1999.

[187] M. Wildt, Ein konstruktivistischer Blick auf Mathematikunterricht, Pädagogik, Heft 7-8/98, 1998, 48-51.

[188] D. Williams, Assessment of open-ended work in the secondary school, in: Evaluation and Assessment in Mathematics Education, Science and Technology Education, Document Series 32, (D. F. Robitaille, ed.), Unesco, Paris, 1989, 135-140.

[189] H. Winter, Kreatives Denken im Sachrechnen, Grundschule 9, no. 3, 1977, 106-110.

[190] H. Winter, Entdeckendes Lernen im Mathematikunterricht, Grundschule 16, no. 4, 1984, 26-29.

[191] H. Winter, Entdeckendes Lernen im Mathematikunterricht. - Einblicke in die Ideengeschichte und ihre Bedeutung für die Pädagogik, Vieweg, Braunschweig, 1989.

[192] H. Winter, Perspektiven eines kreativen Mathematikunterrichts in der allgemeinbildenden Schule - das Wechselspiel von Gestalt und Zahl als heuristische Leitidee, in: Kreatives Denken und Innovation in mathematischen Wissenschaften, B. Zimmermann et al. (Hrsg.), Jenaer Schriften zur Mathematik und Informatik, Universität Jena, 1999.

[193] E. C. Wittmann, Grundfragen der Mathematikdidaktik, Vieweg, Braunschweig, 1980.

[194] E. C. Wittmann und G. N. Müller, Handbuch der produktiven Rechenübungen Band 1, Klett, Stuttgart, 1990.

[195] E. C. Wittmann und G. N. Müller, Handbuch der produktiven Rechenübungen Band 2, Klett, Stuttgart, 1992.

[196] E. C. Wittmann, Mathematisches Denken bei Vor- und Grundschulkindern: eine Einführung in psychologisch-didaktische Experimente, Vieweg, Braunschweig, 1982.

[197] E. C. Wittmann (ed.), Drawing on richness of elementary mathematics in designing substantial learning environments, PME 25, Freundenthal-Institute, Utrecht, 2001.

[198] J. Wittmann, Theorie und Praxis eines ganzheitlichen, analytisch-synthetischen Unterrichts in Grundschule, Hilfsschule, Volksschule, 2. sehr erw. Auflage, Müller \& Kiepenheuer, Potsdam, 1929, (4. Auflage, Crüwell, Dortmund, 1967).

[199] Wong, Investigating Conceptions of Mathematics by the Use of Open-ended Mathematical Problems, A paper submitted in the Topic Study Group 11 at the ICME-9 conference in Makuhari/Tokyo, 2000. 


$$
\text { "graumann" — 2007/8/21 — 13:22 — page } 291 \text { — \#41 }
$$

$\underline{\text { Problemorientierung im Mathematikunterricht - ein Gesichtspunkt d. Qualitätssteigerung } 291}$

[200] T. Wood, Learning to teach mathematics differently: reflection matters, in: Proceedings of the PME-25 conference, Vol. 4, (M. v. d. Heuvel-Panhuizen, ed.), Freudenthal Institute, Utrecht, 2001, 431-438.

[201] B. Zimmermann, Denkprozesse beim Lösen mathematischer Deduktionsaufgaben - Bericht über eine explorative Untersuchung, Journal für Mathematikdidaktik 82, no. 3/(3/4), 1982, 175-206.

[202] B. Zimmermann, From Problem Solving to Problem Finding in Mathematics Instruction, in: Mathematics Education Research in Finland, Yearbook 1985, (P. Kupari, ed.), University of Jyväskylä, Institute for Educational Research, 1986, 81-103, Publication Series B: Theory into Practice 3.

[203] B. Zimmermann, Kaleidoskop elementar-mathematischen Entdeckens. Texte zur mathematischen Forschung und Lehre 1., Franzbecker, Hildesheim, 1995.

[204] B. Zimmermann, Vorstellungen über Mathematik im Wandel ihrer Geschichte und in verschiedenen Kulturen, Beiträge zum Mathematikunterricht 1999, Franzbecker, Hildesheim, 1999a.

[205] B. Zimmermann (Hrsg.), Kreatives Denken und Innovationen in mathematischen Wissenschaften, Jenaer Schriften zur Mathematik und Informatik Math/ Inf/99/29, Friedrich-Schiller-Universität Jena, 1999b.

[206] B. Zimmermann, Darstellungswechsel als wichtige Methode zur Lösung von Problemen - schon in der Geschichte der Mathematik, Der Mathematikunterricht 49, no. $6,2003,6-15$.

PROF. DR. GÜNTER GRAUMANN

UNIVERSITÄT BIELEFELD

DEUTSCHLAND

E-mail: graumann@mathematik.uni-bielefeld.de

PROF. DR. ERKKI PEHKONEN

UNIVERSITÄT HELSINKI

FINNLAND

E-mail: erkki.pehkonen@helsinki.fi

(Received June, 2006) 